\title{
Synthesis of three tetrasaccharides containing 3-O-methyl- D-mannose, as model compounds for xylose-containing carbohydrate chains from $\mathrm{N}$-glycoproteins
}

\author{
Jos G.M. van der Ven, Jac C.H.M. Wijkmans, Johannis P. Kamerling * \\ and Johannes F.G. Vliegenthart \\ Bijvoet Center, Department of Bio-Organic Chemistry, Utrecht University, P.O. Box 80.075, \\ NL-3508 TB Utrecht (Netherlands)
}

(Received April 30th, 1993; accepted July 6th, 1993)

\begin{abstract}
The synthesis is reported of methyl 3,6-di- $O$-(3- $O$-methyl- $\alpha$-D-mannopyranosyl)-2-O- $\beta$-Dxylopyranosyl- $\beta$-D-mannopyranoside (2), methyl $6-O-\alpha$-D-mannopyranosyl-3- $O$-(3- $O$-methyl- $\alpha$-D-mannopyranosyl)-2- $O-\beta$-D-xylopyranosyl- $\beta$-D-mannopyranoside (3), and methyl 3-O- $\alpha$-D-mannopyranosyl-6$O$-(3-O-methyl- $\alpha$-D-mannopyranosyl)-2- $O-\beta$-D-xylopyranosyl- $\beta$-D-mannopyranoside (4). The various methyl $\beta$-D-Man $p$ acceptor derivatives were prepared from the corresponding methyl $\beta$-D-Glc $p$ derivatives via oxidation-reduction. All glycosyl donors were coupled using the trichloroacetimidate method at $-40^{\circ} \mathrm{C}$ in dichloromethane with trimethylsilyl triflate as a catalyst. Methyl-3-O-benzyl-4,6$O$-benzylidene- $\beta$-D-mannopyranoside (7) was condensed with 2,3,4-tri- $O$-acetyl- $\alpha$-D-xylopyranosyl trichloroacetimidate (8). Regioselective reductive 4,6-O-benzylidene ring-opening on the resulting disaccharide derivative, followed by acetylation, and hydrogenation gave methyl 4-O-acetyl-2-O-(2,3,4tri- $O$-acetyl- $\beta$-D-xylopyranosyl)- $\beta$-D-mannopyranoside (12). Coupling of 12 with 2,4,6-tri- $O$-acetyl-3- $O$ methyl- $\alpha$-D-mannopyranosyl trichloroacetimidate (18) afforded tetrasaccharide derivative 19, and subsequent $O$-deacetylation gave 2. Methyl 3-O-benzyl-4,6-O-prop-2-enylidene- $\beta$-D-mannopyranoside (22) was condensed with 2,3,4-tri- $O$-acetyl- $\alpha$-D-xylopyranosyl trichloroacetimidate (8). Regioselective reductive 4,6-O-prop-2-enylidene ring-opening on the resulting disaccharide derivative, followed by acetylation, and deallylation at O-6 gave methyl 4-O-acetyl-3-O-benzyl-2-O-(2,3,4-tri-O-acetyl- $\beta$-Dxylopyranosyl)- $\beta$-D-mannopyranoside (26-a), which was either condensed with $2,3,4,6$-tetra- $O$-acetyl- $\alpha$ D-mannopyranosyl trichloroacetimidate (27) or 18, to give trisaccharide derivatives 28 or 31, respectively. Debenzylation of 28 followed by condensation with 18 gave, after $O$-deacetylation, 3 , whereas debenzylation of $\mathbf{3 1}$ followed by condensation with 27 gave, after $O$-deacetylation, 4 .
\end{abstract}

\section{INTRODUCTION}

Methylated monosaccharide residues only rarely occur in $\mathrm{N}$-linked carbohydrate chains of glycoproteins. So far, 3-O-methyl-D-mannose has been found in hemocyanin of Lymnaea stagnalis ${ }^{1,2}$ and in $\mathrm{N}$-glycoproteins of the dimorphic fungus

* Corresponding author. 
Mucor rouxii ${ }^{3}$. In the hemocyanin, 3-O-methyl- $\alpha$-D-mannose forms an integral part of the core element which also contains a $\beta$-D-xylose residue $(1 \rightarrow 2)$-linked to $\beta$-D-mannose ${ }^{1,2,4}$, whereas novel extensions, including the occurrence of 3-Omethyl- $\beta$-D-galactose, were established ${ }^{2}$ (1).

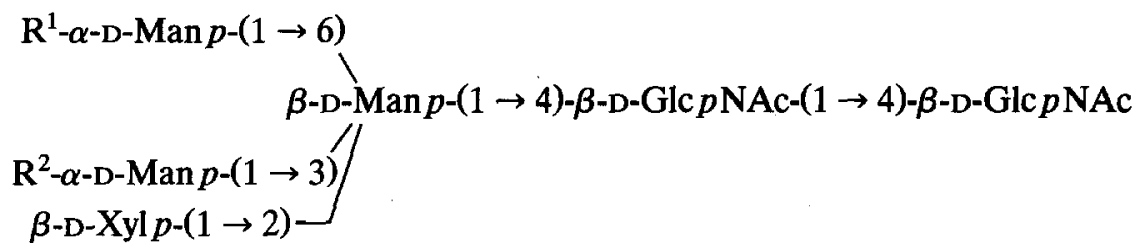

(i) $\mathrm{R}^{1}=\mathrm{R}^{2}=3-O-\mathrm{Me}$

(ii) $\mathbf{R}^{1}=3-O-\mathrm{Me}-\beta$-D-Gal $p$ - $(1 \rightarrow 3)-\beta$-D-Gal $p$ NAc- $(1 \rightarrow 4)-\beta$-D-Glc $p$ NAc- $(1 \rightarrow 2)$ and $\mathbf{R}^{2}=3-O-M e$

(iii) $\mathrm{R}^{1}=\mathbf{H}$ and $\mathrm{R}^{2}=3-O-\mathrm{Me}-\beta$-D-Gal $p$ - $(1 \rightarrow 3)-\beta$-D-Gal $p$ NAc- $(1 \rightarrow 4)-\beta$-D-Glc $p$ NAc- $(1 \rightarrow 2)$

(iv) $\quad \mathbf{R}^{1}=\mathbf{R}^{2}=3$-O-Me- $\beta$-D-Gal $p$ - $(1 \rightarrow 3)-\beta$-D-Gal $p$ NAc- $(1 \rightarrow 4)-\beta$-D-Glc $p$ NAc- $(1 \rightarrow 2)$

(v) $\quad \mathbf{R}^{1}=\alpha$-L-Fuc $p$ - $(1 \rightarrow 2)-\beta$-D-Gal $p$ - $(1 \rightarrow 3)-\beta$-D-Gal $p$ NAc- $(1 \rightarrow 4)-\beta$-D-Glc $p$ NAc- $(1 \rightarrow 2)$ and $\mathbf{R}^{2}=3-O-M e$

(vi) $\mathbf{R}^{1}=\mathbf{H}$ and $\mathrm{R}^{2}=\alpha$-L-Fucp- $(1 \rightarrow 2)-\beta$-D-Gal $p$ - $(1 \rightarrow 3)-\beta$-D-Gal $p$ NAc- $(1 \rightarrow 4)-\beta$-D-Glc $p$ NAc- $(1 \rightarrow 2)$

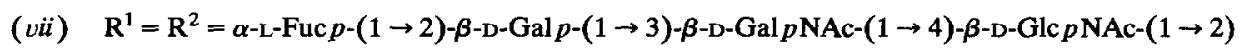

(viii) $\mathrm{R}^{1}=3-O-\mathrm{Me}-\beta$-D-Galp- $(1 \rightarrow 3)-\beta$-D-Gal $p$ NAc- $(1 \rightarrow 4)-\beta$-D-Glc $p$ NAc- $(1 \rightarrow 2)$ and $\mathrm{R}^{2}=\alpha$-L-Fuc $p-(1 \rightarrow 2)-\beta$-D-Gal $p-(1 \rightarrow 3)-\beta$-D-Gal $p$ NAc- $(1 \rightarrow 4)-\beta$-D-Glc $p$ NAc- $(1 \rightarrow 2)$

As a part of our program concerning the biosynthesis and conformation analysis of these types of glycans ${ }^{5-7}$, the synthesis of some related oligosaccharide elements has been reported ${ }^{8,9}$. Here, we describe the synthesis of the tetrasaccharides 2,3 , and 4, containing either one or two 3-O-methyl- $\alpha$-D-mannose residues, representing the branching part of the core structure in which the $\beta$-D-mannose configuration is mimicked as a methyl glycoside. It should be noted that $\mathbf{4}$ is not a structural element of $L$. stagnalis hemocyanin.

$$
\begin{array}{cl}
\mathrm{R}^{1}-\alpha-\mathrm{D}-\mathrm{Man} p-(1 \rightarrow 6) & 2 \mathrm{R}^{1}=\mathrm{R}^{2}=3-O-\mathrm{Me} \\
\beta \text {-D-Man } p-(1 \rightarrow \mathrm{OMe} & 3 \mathrm{R}^{1}=\mathrm{H}, \mathrm{R}^{2}=3-O-\mathrm{Me} \\
\mathrm{R}^{2}-\alpha \text {-D-Man } p-(1 \rightarrow 3) & 4 \mathrm{R}^{1}=3-O-\mathrm{Me}, \mathrm{R}^{2}=\mathrm{H} \\
\beta \text {-D-Xyl } p-(1 \rightarrow 2) &
\end{array}
$$

\section{RESULTS AND DISCUSSION}

For the synthesis of tetrasaccharide 2, methyl 4-O-acetyl-2-O-(2,3,4-tri-O-acetyl$\beta$-D-xylopyranosyl)- $\beta$-D-mannopyranoside (12) and 2,4,6-tri- $O$-acetyl-3- $O$-methyl- $\alpha$ D-mannopyranosyl trichloroacetimidate (18) where chosen as building blocks. The 
preparation of methyl $\beta$-D-Man $p$, or $\beta$-D-mannopyranosides in general, can be accomplished via 'direct' or 'indirect' methods ${ }^{8-10}$. Because the synthesis of xylose-containing carbohydrate chains using the indirect method via oxidation-reduction has proved to be very convenient in our hands ${ }^{8,9}$, this strategy was also followed in the present report. Thus, methyl 3- $O$-benzyl- $\beta$-D-glucopyranoside ${ }^{11}$ was benzylidenated using $\alpha, \alpha$-dimethoxytoluene and $p$-toluenesulfonic acid to give crystalline 5 (75\%). Oxidation of 5 using 2:1 dimethyl sulfoxide-acetic anhydride gave 6 and a small amount of a by-product. Evaporation of the reagents followed by treatment of the residue with sodium borohydride afforded 7 (66\% from 5) and some 5 (10\% from 5$)$. The by-product in the oxidation of 5 , probably methyl 3- $O$-benzyl-4,6- $O$-benzylidene-2- $O$-methylthiomethyl- $\beta$-D-glucopyranoside ${ }^{12}$, could easily be separated from the reaction products after the reduction. Its amount can be minimized by using acetic anhydride free from acetic acid ${ }^{13}$. Stereoselective xylosylation of 7 proceeded satisfactorily using 2,3,4-tri- $O$-acetyl- $\alpha$-D-xylopyranosyl trichloroacetimidate $(8)^{9,14}$ in dichloromethane at $-40^{\circ} \mathrm{C}$, with trimethylsilyl triflate as a catalyst, to give exclusively the $\beta$-linked disaccharide derivative 9 in $75 \%$ yield. Regioselective reductive ring-opening of the 4,6-benzylidene acetal, using sodium cyanoborohydride and hydrochloric acid in tetrahydrofuran ${ }^{15}(\rightarrow 10,67 \%)$, followed by treatment with pyridine-acetic anhydride $(\rightarrow 11,99 \%)$ and subsequent hydrogenation using $10 \% \mathrm{Pd}-\mathrm{C}$ gave 12 in $90 \%$ yield.

The synthesis of the 3-O-methyl-D-mannose donor (18) involved also the application of the oxidation-reduction method, whereby benzyl 4,6-O-isopropylidene-3$O$-methyl- $\beta$-D-glucopyranoside (13) was converted into the corresponding manno derivative (15). Isopropylidenation of benzyl 3- $O$-methyl- $\beta$-D-glucopyranoside ${ }^{16}$, using 2,2-dimethoxypropane and $p$-toluenesulfonic acid, yielded crystalline 13 $(88 \%)$, which was oxidised using 2:1 dimethyl sulfoxide-acetic anhydride to give 14 in its keto and gem-diol form (79\%), as shown by ${ }^{13} \mathrm{C}$ NMR spectroscopy. Reduction of this mixture with sodium borohydride afforded $15(79 \%)$ and some $13(7 \%)$. Then compound 15 was de-isopropylidenated and acetylated $(\rightarrow \mathbf{1 6}$, 93\%), hydrogenolysed $(\rightarrow \mathbf{1 7}, 97 \%)$, and treated with trichloroacetonitrile in the presence of 1,8-diazabicyclo[5.4.0]undec-7-ene ${ }^{17}$ to afford $18(73 \%)$. Coupling of 18 with 12 in dichloromethane $\left(1 \mathrm{~h},-40^{\circ} \mathrm{C}\right)$, using trimethylsilyl triflate as a catalyst, gave tetrasaccharide derivative 19 in $71 \%$ yield. Finally, Zemplén deacetylation of 19 afforded $2(90 \%)$. For ${ }^{1} \mathrm{H}$ NMR structural-reported-group data, see Table I.

For the synthesis of the tetrasaccharides 3 and 4, having a 3-O-methyl- $\alpha$-D-mannose residue linked either $1 \rightarrow 3$ or $1 \rightarrow 6$, the 4,6-benzylidene acetal in compound 9 was changed for a 4,6-(prop-2-enylidene) acetal ${ }^{18}$. Regioselective reductive ring-opening and acetylation gives a synthon with an allyl group at O-6 and a benzyl group at O-3, making the regiospecific introduction of 3-O-methyl- $\alpha$-D-mannose and $\alpha$-D-mannose residues possible. To this end, methyl 3- $O$-benzyl- $\beta$-D-glucopyranoside $^{11}$ was treated with acrolein dimethyl acetal and $p$-toluenesulfonic acid in $N, N$-dimethylformamide ${ }^{18}$ to give crystalline $20(75 \%)$. Oxidation of 20 using 2:1 dimethyl sulfoxide-acetic anhydride gave 21, in its keto and gem-diol 


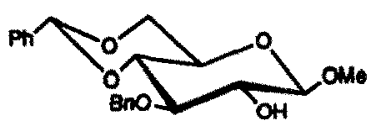

5

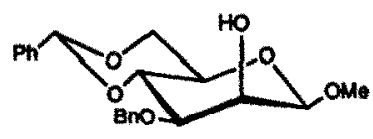

7
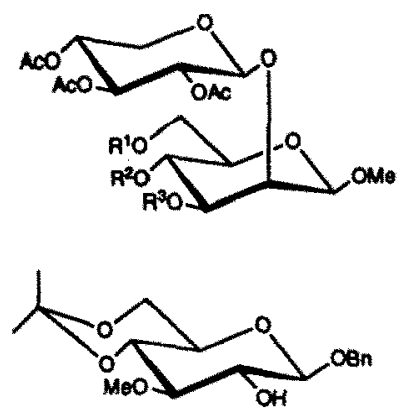

13

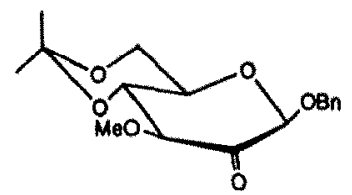

14

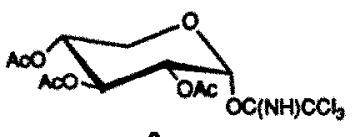

- $R^{1}, R^{2}-C H P h_{4} R^{3}=B n$

10 $R^{\prime}=R^{3}=8 n, A^{2}=H$

$11 R^{1}-R^{3}=B n, R^{2}=A C$

$12 R^{1}=R^{3}=H_{k} R^{2}=A C$

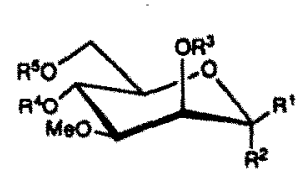

$15 R^{*}=O n_{1} R^{2}-A^{3}-H_{1} A^{4}, R^{6}=C\left(C_{b}\right)_{2}$

$16 A^{\prime}=O B n, A^{2}=H, A^{3}-A^{4}=A^{5}=A C$

$17 R^{1}, R^{2}=H, O H, R^{3}=A^{4}=R^{5}=A C$

16 $R^{1}-H, R^{2}=O C(N H) C_{3} A^{3}=A^{4}=A^{3}=A C$

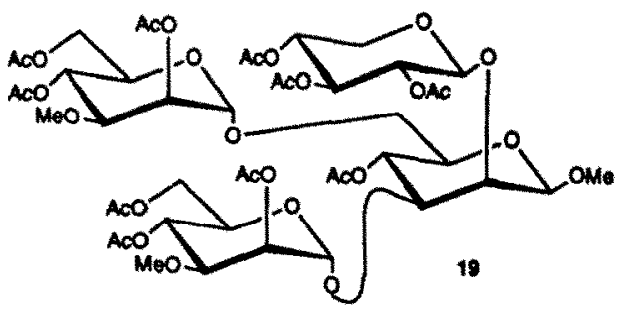




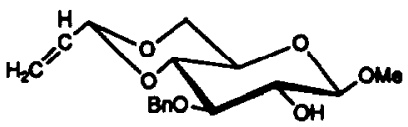

20
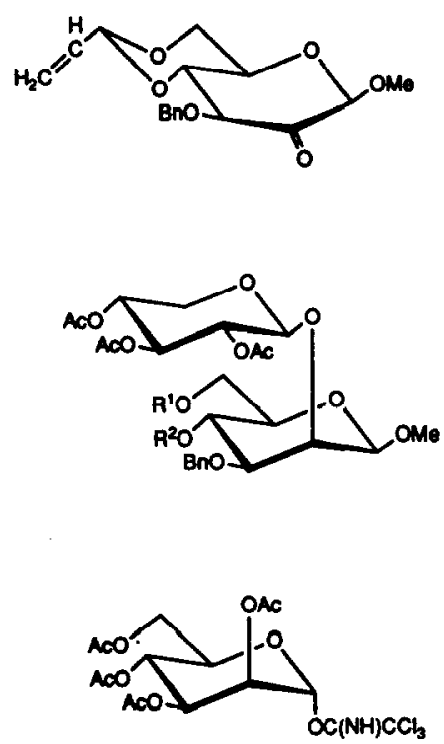

27

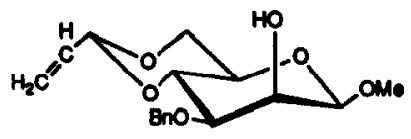

22

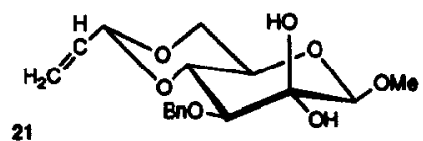

form. After evaporation of the reagents, the residue, still containing a small amount of by-product (see above), was directly treated with sodium borohydride to afford 22 (56\% from 20$)$ and 20 (11\% from 20). Xylosylation of 22 with 8 in dichloromethane at $-40^{\circ} \mathrm{C}$, using trimethylsilyl triflate as a catalyst, gave $23(89 \%)$. Regioselective reductive ring-opening of the 4,6-(prop-2-enylidene) acetal, using sodium cyanoborohydride and hydrochloric acid in tetrahydrofuran $(\rightarrow 24,69 \%)$, followed by treatment with pyridine-acetic anhydride gave 25 (98\%). De-allylation of 25 using the Wilkinson catalyst in the presence of 1,4-diazabicyclo[2.2.2]octane ${ }^{19}$ followed by hydrolysis yielded one spot in TLC (26-a). However, during column chromatography, acetyl migration in the $\beta$-D-mannose residue occurred from $\mathrm{O}-4 \rightarrow \mathrm{O}-6$, and the $4-\mathrm{O}$-acetyl derivative $26-\mathrm{a}$ could be isolated in only $53 \%$ yield, together with the undesired 6-O-acetyl derivative 26-b (40\%). This problem could be overcome by direct glycosylation after work-up, as is shown for the synthesis of 31.

Condensation of compound 26-a with 27 in dichloromethane $\left(1 \mathrm{~h},-40^{\circ} \mathrm{C}\right)$, using trimethylsilyl triflate as a catalyst $(\rightarrow \mathbf{2 8}, \mathbf{7 7 \%}$ ), followed by hydrogenolysis of the benzyl ether of the resulting trisaccharide derivative gave acceptor 29 (98\%), which was mannosylated with 18 in dichloromethane (trimethylsilyl triflate, $1.5 \mathrm{~h}$, 
TABLE I

$300-\mathrm{MHz}^{1} \mathrm{H}$ NMR data of the tetrasaccharides $2-4$, together with those of reference compound methyl 3,6-di-O- $\alpha$-D-mannopyranosyl-2- $O-\beta$-D-xylopyranosyl- $\beta$-D-mannopyranoside ${ }^{8}$ (R)

\begin{tabular}{|c|c|c|c|c|c|}
\hline \multirow[t]{2}{*}{ Residue } & \multirow{2}{*}{$\begin{array}{l}\text { Reporter } \\
\text { group }(J)\end{array}$} & \multicolumn{4}{|c|}{$\delta(\mathrm{ppm}) / J(\mathrm{~Hz})$} \\
\hline & & 2 & 3 & 4 & $\mathbf{R}$ \\
\hline$\beta$-Man & $\begin{array}{l}\mathrm{H}-1^{a} \\
\mathrm{H}-2\left(J_{2,3}\right) \\
\mathrm{CH}_{3} \mathrm{O}\end{array}$ & $\begin{array}{l}4.689 \\
4.203(2.3) \\
3.532\end{array}$ & $\begin{array}{l}4.682 \\
4.197(2.1) \\
3.526\end{array}$ & $\begin{array}{l}4.687 \\
4.200(2.3) \\
3.531\end{array}$ & $\begin{array}{l}4.681 \\
4.197(2.8) \\
3.523\end{array}$ \\
\hline Xyl & $\mathrm{H}-1\left(J_{1,2}\right)$ & $4.488(7.4)$ & $4.482(7.4)$ & $4.483(7.4)$ & $4.474(7.5)$ \\
\hline$\alpha-\operatorname{Man}-(1 \rightarrow 6)$ & $\begin{array}{l}\mathrm{H}-1\left(J_{1,2}\right) \\
\mathrm{H}-2\left(J_{2,3}\right) \\
\mathrm{CH}_{3} \mathrm{O}\end{array}$ & $\begin{array}{l}4.948(1.8) \\
4.263(3.2) \\
3.459\end{array}$ & $\begin{array}{l}4.903(1.7) \\
\text { n.d. }{ }^{b}\end{array}$ & $\begin{array}{l}4.945(1.8) \\
4.258(3.0) \\
3.457\end{array}$ & $\begin{array}{l}4.900(1.8) \\
4.010(3.5)\end{array}$ \\
\hline$\alpha-\operatorname{Man}-(1 \rightarrow 3)$ & $\begin{array}{l}\mathrm{H}-1\left(J_{1,2}\right) \\
\mathrm{H}-2\left(J_{2,3}\right) \\
\mathrm{CH}_{3} \mathrm{O}\end{array}$ & $\begin{array}{l}5.183(1.5) \\
4.303(2.9) \\
3.445\end{array}$ & $\begin{array}{l}5.180(1.5) \\
4.294 \text { (n.d.) } \\
3.445\end{array}$ & $\begin{array}{l}5.141(1.4) \\
4.043(3.3)\end{array}$ & $\begin{array}{l}5.138(1.8) \\
4.035(3.4)\end{array}$ \\
\hline
\end{tabular}

${ }^{a}$ Because of the very small $J_{1,2}$ value, $\mathrm{H}-1$ is observed as a singlet. ${ }^{b}$ Not determined.

$\left.-40^{\circ} \mathrm{C}\right)$ to afford tetrasaccharide derivative $30(61 \%)$. Zemplén deacetylation then gave compound $3(96 \%)$. Trisaccharide derivative 31 was prepared in $51 \%$ yield by condensation of non-purified 26-a (see above) with 18 in dichloromethane, using trimethylsilyl triflate as a catalyst $\left(1 \mathrm{~h},-40^{\circ} \mathrm{C}\right)$. Hydrogenolysis of $\mathbf{3 1}(\rightarrow \mathbf{3 2}, 98 \%)$ and subsequent glycosylation of 32 with 27 gave tetrasaccharide derivative 33 (70\%), which after Zemplén deacetylation afforded 4 (96\%). For ${ }^{1} \mathrm{H}$ NMR structural-reporter-group data of 3 and 4, see Table I.
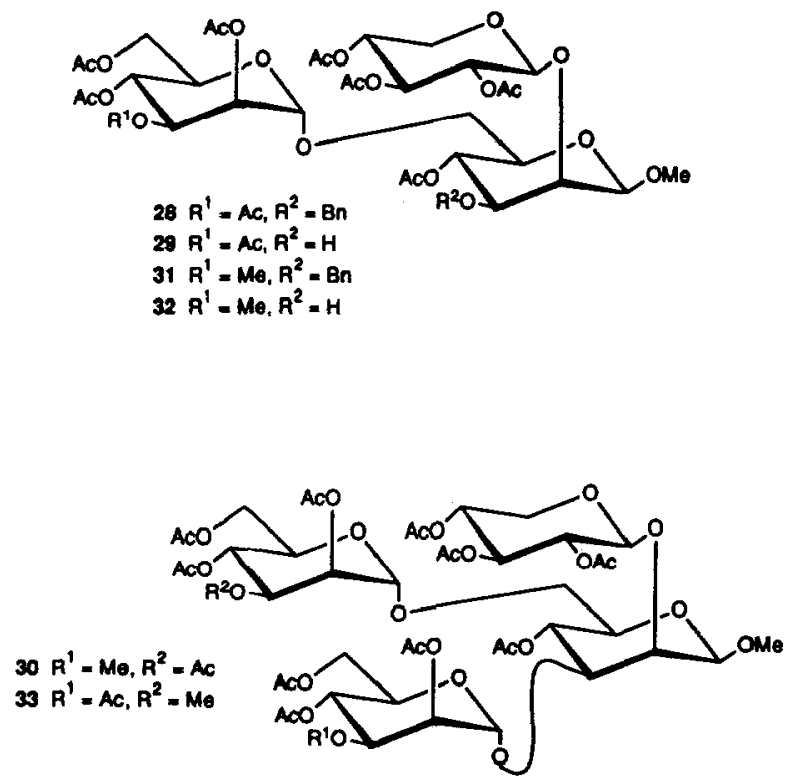


\section{TABLE II}

Coupling constants $(J, \mathrm{~Hz})$ between the skeleton protons of the xylose residue in protected oligosaccharides synthesised in this study

\begin{tabular}{lllllll}
\hline Compound & $J_{1,2}$ & $J_{2,3}$ & $J_{3,4}$ & $J_{4,5 e q}$ & $J_{4,5 a x}$ & $J_{5,5}$ \\
\hline $\mathbf{9}$ & 6.1 & 8.1 & 7.9 & 4.7 & 7.5 & -12.0 \\
$\mathbf{1 0}$ & 6.3 & 8.0 & 8.0 & 4.8 & 8.0 & -11.9 \\
$\mathbf{1 2}$ & 6.9 & n.d. ${ }^{a}$ & n.d. & 5.1 & n.d. & -11.8 \\
$\mathbf{1 9}$ & 7.1 & 9.1 & n.d. & 5.6 & 9.0 & -12.3 \\
$\mathbf{2 3}$ & 5.6 & 7.7 & 7.7 & 4.6 & 7.4 & -12.0 \\
$\mathbf{2 4}$ & 6.3 & 8.2 & 8.0 & 4.8 & 8.0 & -12.9 \\
$\mathbf{2 5}$ & n.d. & n.d. & n.d. & 3.4 & n.d. & -12.0 \\
$\mathbf{2 6 - a}$ & n.d. & n.d. & n.d. & 3.5 & 4.7 & -12.4 \\
$\mathbf{2 6 - b}$ & 7.0 & 9.0 & 8.8 & 5.1 & 8.8 & -11.8 \\
$\mathbf{2 8}$ & n.d. & n.d. & n.d. & 3.5 & 4.9 & -12.3 \\
$\mathbf{2 9}$ & 6.7 & 8.6 & n.d. & n.d. & 8.8 & -11.9 \\
$\mathbf{3 0}$ & n.d. & n.d. & n.d. & 5.2 & 8.2 & -11.6 \\
$\mathbf{3 1}$ & 4.7 & 6.5 & n.d. & 5.5 & n.d. & -12.3 \\
$\mathbf{3 3}$ & 5.8 & n.d. & 7.1 & 4.4 & 7.1 & n.d. \\
\hline
\end{tabular}

${ }^{a}$ Not determined.

Evaluation of the ${ }^{1} \mathrm{H}$ NMR spectra of the compounds containing a protected $\beta$-D-xylopyranose residue showed that the $J$ values between the skeleton protons vary to some extent (Table II). Taking into account especially the $J_{1,2}, J_{4,5 a x}$, and $J_{4,5 e q}$ values, it can be concluded that these variations have to reflect changes in conformation of the xylose residue. This behaviour is not uncommon for protected $\beta$-D-Xyl $p$ derivatives, and quite often ${ }^{1} C_{4}(\mathrm{D}) \Leftrightarrow{ }^{4} C_{1}(\mathrm{D})$ conformational equilibria are suggested in case of small $J_{1,2} / J_{4,5 a x} / J_{4, \text { Seq }}$ values, explained by the anomeric effect which balances the destabilising effect of the three axially oriented $O$-acetyl groups on the Xyl $p$ ring $^{9,20-26}$. In this context it has to be mentioned that distortions of the ${ }^{4} C_{1}(\mathrm{D})$ chair can also lead to deviating $J$ values.

\section{EXPERIMENTAL}

General methods. - The ${ }^{1} \mathrm{H}(300$ and $360 \mathrm{MHz})$ and ${ }^{13} \mathrm{C}$ (APT, $50 \mathrm{MHz}$ ) NMR spectra were recorded at $25^{\circ} \mathrm{C}$ with a Bruker AC 300, WP 200, or HX 360 spectrometer. Chemical shifts $(\delta)$ are given in ppm relative to the signal for internal $\mathrm{Me}_{4} \mathrm{Si}\left(\mathrm{CDCl}_{3}\right)$ or sodium 4,4-dimethyl-4-silapentane-1-sulfonate $\left(\mathrm{D}_{2} \mathrm{O}\right.$; indirectly to internal acetone, $\delta 2.225$ ) for ${ }^{1} \mathrm{H}$, and to the signal for internal $\mathrm{Me}_{4} \mathrm{Si}$ $\left(\mathrm{CDCl}_{3}\right.$; indirectly to $\left.\mathrm{CDCl}_{3}, \delta 76.9\right)$ or external $\mathrm{Me}_{4} \mathrm{Si}\left(\mathrm{D}_{2} \mathrm{O}\right.$; indirectly to internal acetone, $\delta 31.55$ ) for ${ }^{13} \mathrm{C}$. Column chromatography was performed on Kieselgel 60 (Merck, $<230$ mesh) and fractions were monitored by TLC on Kieselgel $60 \mathrm{~F}_{254}$ (Merck) by detection with $\mathrm{UV}$ light and then charring with $\mathrm{H}_{2} \mathrm{SO}_{4}$. Optical rotations were measured for solutions in $\mathrm{CHCl}_{3}$, unless otherwise stated, at $20^{\circ} \mathrm{C}$ with a Perkin-Elmer 241 polarimeter, using a $10-\mathrm{cm} 1-\mathrm{mL}$ cell. Melting points 
were determined with a Mettler FP 51 instrument. In the work-up procedures, washings were carried out three times with appropriate quantities of water or aq $5 \% \mathrm{NaHCO}_{3}$ unless indicated otherwise. Solvents were evaporated under reduced pressure at $40^{\circ} \mathrm{C}$ (bath) unless indicated otherwise. All solvents were distilled from the appropriate drying agents.

Methyl 3-O-benzyl-4,6-O-benzylidene- $\beta$-D-glucopyranoside (5). - To a solution of methyl 3-O-benzyl- $\beta$-D-glucopyranoside ${ }^{11}(1.8 \mathrm{~g}, 6.3 \mathrm{mmol})$ in $N, N$-dimethylformamide $(17 \mathrm{~mL})$ and $\alpha, \alpha$-dimethoxytoluene $(1.8 \mathrm{~mL}, 12 \mathrm{mmol})$ was added $p$ toluenesulfonic acid monohydrate $(150 \mathrm{mg})$. After stirring for $1 \mathrm{~h}$ under reduced pressure (20 mmHg), TLC (95:5 $\mathrm{CH}_{2} \mathrm{Cl}_{2}$-acetone) indicated the reaction to be complete, and solid $\mathrm{NaHCO}_{3}$ was added. The mixture was diluted with $\mathrm{CH}_{2} \mathrm{Cl}_{2}$ $(300 \mathrm{~mL})$, washed with water, dried $\left(\mathrm{MgSO}_{4}\right)$, filtered, and concentrated. The residue was crystallised from EtOH to give $5(1.8 \mathrm{~g}, 75 \%) ; \mathrm{mp} 181^{\circ} \mathrm{C} ;[\alpha]_{\mathrm{D}}-38^{\circ}(\mathrm{c}$ 1); $R_{f} 0.60 ;$ lit $^{27} \mathrm{mp} 182-183^{\circ} \mathrm{C} ;[\alpha]_{\mathrm{D}}-51^{\circ}\left(\mathrm{c} 0.86, \mathrm{CHCl}_{3}\right)$. NMR data $\left(\mathrm{CDCl}_{3}\right)$ : ${ }^{1} \mathrm{H}, \delta$ 7.48-7.25 (m, $\left.10 \mathrm{H}, 2 \mathrm{Ph}\right), 5.576(\mathrm{~s}, 1 \mathrm{H}, \mathrm{PhCH}), 4.974$ and $4.792(2 \mathrm{~d}$, each 1 $\mathrm{H}, \mathrm{PhCH} \mathrm{H}_{2} \mathrm{O}$ ), 4.364 (dd, $\left.1 \mathrm{H}, J_{6 \mathrm{a}, 5} 5.0, J_{6 \mathrm{a}, 6 \mathrm{~b}}-10.5 \mathrm{~Hz}, \mathrm{H}-6 \mathrm{a}\right), 4.328\left(\mathrm{~d}, 1 \mathrm{H}, J_{1,2}\right.$ $7.6 \mathrm{~Hz}, \mathrm{H}-1), 3.804\left(\mathrm{t}, 1 \mathrm{H}, J_{4,3}=J_{4,5}=10.2 \mathrm{~Hz}, \mathrm{H}-4\right), 3.572\left(\mathrm{~s}, 3 \mathrm{H}, \mathrm{CH}_{3} \mathrm{O}\right), 2.442$ $\left(\mathrm{d}, 1 \mathrm{H}, J_{\mathrm{HO}-2,2} 2.2 \mathrm{~Hz}, \mathrm{HO}-2\right) ;{ }^{13} \mathrm{C}, \delta 137.1,128.8-127.7$, and $125.9\left(C_{6} \mathrm{H}_{5} \mathrm{CH}_{2} \mathrm{O}\right.$ and $\left.\mathrm{C}_{6} \mathrm{H}_{5} \mathrm{CHO}\right), 104.1(\mathrm{PhCH}), 101.1(\mathrm{C}-1), 81.3,80.1,74.1$, and $66.2(\mathrm{C}-2,3,4,5)$, 74.5 and 68.6 $\left(\mathrm{PhCH}_{2} \mathrm{O}\right.$ and $\left.\mathrm{C}-6\right), 57.3\left(\mathrm{CH}_{3} \mathrm{O}\right)$. Anal. Calcd for $\mathrm{C}_{21} \mathrm{H}_{24} \mathrm{O}_{6}$ : $\mathrm{C}$, $67.73 ; \mathrm{H}, 6.50$. Found: $\mathrm{C}, 67.67 ; \mathrm{H}, 6.65$.

Methyl 3-O-benzyl-4,6-O-benzylidene- $\beta$-D-mannopyranoside (7).-A solution of 5 $(1.2 \mathrm{~g}, 3.2 \mathrm{mmol})$ in $1: 2$ acetic anhydride- $-\mathrm{Me}_{2} \mathrm{SO}(22 \mathrm{~mL})$ was stirred for $20 \mathrm{~h}$. TLC (95:5 $\mathrm{CH}_{2} \mathrm{Cl}_{2}$-acetone) then showed the ulose $6\left(R_{f} 0.50-0.72\right)$ and a faster moving product $\left(R_{f} 0.93\right)$. The mixture was concentrated, and toluene $(3 \times 15 \mathrm{~mL})$ was evaporated from the residue, which was then further concentrated in vacuo (bath $\left.<60^{\circ} \mathrm{C}\right)$ to yield 6 , isolated as a yellow solid $(1.2 \mathrm{~g})$. A purified sample gave: $[\alpha]_{\mathrm{D}}-80^{\circ}($ c 1$\left.) ; \mathrm{lit}^{27}[\alpha]_{\mathrm{D}}-81^{\circ}(c) 1, \mathrm{CHCl}_{3}\right)$. NMR data $\left(\mathrm{CDCl}_{3}\right):{ }^{1} \mathrm{H}, \delta$ $7.51-7.30(\mathrm{~m}, 10 \mathrm{H}, 2 \mathrm{Ph}), 5.587(\mathrm{~s}, 1 \mathrm{H}, \mathrm{PhCH}), 4.970$ and $4.764(2 \mathrm{~d}$, each $1 \mathrm{H}$, $\mathrm{PhCH} \mathrm{H}_{2} \mathrm{O}$ ), 4.780 (s, $1 \mathrm{H}, \mathrm{H}-1$ ), 4.460 (dd, $1 \mathrm{H}, J_{6 \mathrm{a}, 5} 4.7, J_{6 \mathrm{a}, 6 \mathrm{~b}}-10.3 \mathrm{~Hz}, \mathrm{H}-6 \mathrm{a}$ ), $4.240\left(\mathrm{dd}, 1 \mathrm{H}, J_{3,4} 10.3 \mathrm{~Hz}, \mathrm{H}-3\right), 3.761(\mathrm{~m}, 1 \mathrm{H}, \mathrm{H}-5), 3.601\left(\mathrm{~s}, 3 \mathrm{H}, \mathrm{CH}_{3} \mathrm{O}\right) ;{ }^{13} \mathrm{C}, \delta$ $188.1(\mathrm{C}-2), 137.1,136.7,129.0-127.8$, and $125.9\left(C_{6} \mathrm{H}_{5} \mathrm{CH}_{2} \mathrm{O}\right.$ and $\left.\mathrm{C}_{6} \mathrm{H}_{5} \mathrm{CHO}\right)$, 101.4 and $100.9(\mathrm{C}-1$ and $\mathrm{PhCH}), 81.9,81.8$, and $66.3(\mathrm{C}-3,4,5), 73.1$ and 68.4 $\left(\mathrm{PhCH}_{2} \mathrm{O}\right.$ and $\left.\mathrm{C}-6\right), 56.9\left(\mathrm{CH}_{3} \mathrm{O}\right)$.

To a solution of $6(1.0 \mathrm{~g}, 2.7 \mathrm{mmol})$ in $1: 1 \mathrm{CH}_{2} \mathrm{Cl}_{2}-\mathrm{MeOH}(20 \mathrm{~mL})$ containing Kieselgel 60 (70-230 mesh, $0.5 \mathrm{~g})$ was added $\mathrm{NaBH}_{4}(0.4 \mathrm{~g}, 11 \mathrm{mmol})$ in portions during $30 \mathrm{~min}$ at $0^{\circ} \mathrm{C}$. After $30 \mathrm{~min}$, TLC $\left(95: 5 \mathrm{CH}_{2} \mathrm{Cl}_{2}\right.$-acetone) showed the formation of $5 \cdot\left(R_{f}, 0.60\right)$ and $7\left(R_{f}, 0.41\right)$ in the ratio $\sim 1: 7$. The mixture was diluted with $\mathrm{CH}_{2} \mathrm{Cl}_{2}(250 \mathrm{~mL})$, filtered, washed with water, dried $\left(\mathrm{MgSO}_{4}\right)$, filtered, and concentrated. Column chromatography $\left(95: 5 \mathrm{CH}_{2} \mathrm{Cl}_{2}\right.$-acetone) of the residue gave $5(100 \mathrm{mg}, 10 \%)$, and then $7(660 \mathrm{mg}, 66 \%)$, both isolated as white solids (yields calculated from 5). Compound 7: $\mathrm{mp} 119^{\circ} \mathrm{C}$ (from EtOH); $[\alpha]_{\mathrm{D}}-28^{\circ}$ (c 1); $\operatorname{lit}^{27} \mathrm{mp} 119-120^{\circ} \mathrm{C}(\mathrm{EtOH}) ;[\alpha]_{\mathrm{D}}-33^{\circ}\left(c \quad 0.86, \mathrm{CHCl}_{3}\right)$. NMR data 
$\left(\mathrm{CDCl}_{3}\right):{ }^{1} \mathrm{H}, \delta$ 7.52-7.20 (m, 10 H, $\left.2 \mathrm{Ph}\right), 5.614(\mathrm{~s}, 1 \mathrm{H}, \mathrm{PhCH}), 4.871$ and $4.777(2$ d, each $1 \mathrm{H}, \mathrm{PhCH} \mathrm{H}_{2} \mathrm{O}$ ), 4.431 (bs, $1 \mathrm{H}, \mathrm{H}-1$ ), 4.344 (dd, $1 \mathrm{H}, J_{6 \mathrm{a}, 5} 4.9, J_{6 \mathrm{a}, 6 \mathrm{~b}}-10.5$ $\mathrm{Hz}, \mathrm{H}-6 \mathrm{a}$ ), 3.657 (dd, $1 \mathrm{H}, J_{3,2} 3.3, J_{3,4} 9.6 \mathrm{~Hz}, \mathrm{H}-3$ ), 3.565 (s, $3 \mathrm{H}, \mathrm{CH}_{3} \mathrm{O}$ ), 3.355 $(\mathrm{m}, 1 \mathrm{H}, \mathrm{H}-5), 2.529$ (d, $\left.1 \mathrm{H}, J_{\mathrm{HO}-2,2} 1.2 \mathrm{~Hz}, \mathrm{HO}-2\right) ;{ }^{13} \mathrm{C}, \delta 137.8,137.3$, 128.8-127.7, and $125.9\left(C_{6} \mathrm{H}_{5} \mathrm{CH}_{2} \mathrm{O}\right.$ and $\left.\mathrm{C}_{6} \mathrm{H}_{5} \mathrm{CH}\right), 101.3(\mathrm{C}-1$ and $\mathrm{PhCH}), 78.3$, 76.5, 69.7, and 66.7 (C-2,3,4,5), 72.4 and $68.4\left(\mathrm{C}-6\right.$ and $\left.\mathrm{PhCH}_{2} \mathrm{O}\right), 57.1\left(\mathrm{CH}_{3} \mathrm{O}\right)$. Anal. Calcd for $\mathrm{C}_{21} \mathrm{H}_{24} \mathrm{O}_{6}$ : C, 67.73; $\mathrm{H}, 6.50$. Found: $\mathrm{C}, 67.48 ; \mathrm{H}, 6.61$.

Methyl 3-O-benzyl-4,6-O-benzylidene-2-O-(2,3,4-tri-O-acetyl- $\beta$-D-xylopyranosyl)$\beta$-D-mannopyranoside (9). - A solution of $7(568 \mathrm{mg}, 1.5 \mathrm{mmol})$ and 2,3,4-tri- $O$ acetyl- $\alpha$-D-xylopyranosyl trichloroacetimidate ${ }^{14}(8 ; 977 \mathrm{mg}, 2.3 \mathrm{mmol})$ in $\mathrm{CH}_{2} \mathrm{Cl}_{2}$ $(10 \mathrm{~mL})$ containing powdered $4 \mathrm{~A}$ molecular sieves $(5.5 \mathrm{~g})$ was stirred for $30 \mathrm{~min}$ under $\mathrm{N}_{2}$. Then a solution of trimethylsilyl triflate $(65 \mu \mathrm{L})$ in $\mathrm{CH}_{2} \mathrm{Cl}_{2}(12 \mathrm{~mL})$ was added dropwise at $-40^{\circ} \mathrm{C}$, and after $2 \mathrm{~h}$, when TLC showed the disappearance of 7 and the formation of $9\left(R_{f} 0.76,95: 5 \mathrm{CH}_{2} \mathrm{Cl}_{2}\right.$-acetone), pyridine $(1 \mathrm{~mL})$ was added. The mixture was filtered through Celite, then concentrated, and toluene $(3 \times 10 \mathrm{~mL})$ was evaporated from the residue. Two-fold column chromatography [first 95:5 $\mathrm{CH}_{2} \mathrm{Cl}_{2}$-acetone, then 1:1 light petroleum (bp 40-60 ${ }^{\circ} \mathrm{C}$ )-EtOAc] of the residue gave 9, isolated as a white foam $(740 \mathrm{mg}, 75 \%) ;[\alpha]_{\mathrm{D}}-96^{\circ}(c 1) ; R_{f}$ 0.46 [1:1 light petroleum (bp $\left.40-60^{\circ} \mathrm{C}\right)$-EtOAc]. NMR data $\left(\mathrm{CDCl}_{3}\right):{ }^{1} \mathrm{H}, \delta$ 7.51-7.26 (m, $10 \mathrm{H}, 2 \mathrm{Ph}$ ), 5.591 (s, $1 \mathrm{H}, \mathrm{PhCH}$ ), 5.189 (t, $1 \mathrm{H}, J_{3^{\prime}, 4^{\prime}} 7.9 \mathrm{~Hz}, \mathrm{H}-3^{\prime}$ ), $5.058\left(\mathrm{dd}, 1 \mathrm{H}, J_{2^{\prime}, 3^{\prime}} 8.1 \mathrm{~Hz}, \mathrm{H}-2^{\prime}\right), 4.933\left(\mathrm{~m}, 1 \mathrm{H}, J_{4^{\prime}, 5^{\prime} \text { eq }} 4.7 \mathrm{~Hz}, \mathrm{H}-4^{\prime}\right), 4.930$ (d, 1 $\left.\mathrm{H}, J_{1^{\prime}, 2^{\prime}} 6.1 \mathrm{~Hz}, \mathrm{H}-1^{\prime}\right), 4.763$ (s, $\left.2 \mathrm{H}, \mathrm{PhCH}_{2} \mathrm{O}\right), 4.315$ (s, $\left.1 \mathrm{H}, \mathrm{H}-1\right), 4.155$ (d, $1 \mathrm{H}$, $J_{2,3} 3.0 \mathrm{~Hz}, \mathrm{H}-2$ ), 3.564 (dd, $1 \mathrm{H}, J_{3,4} 9.9 \mathrm{~Hz}, \mathrm{H}-3$ ), $3.462\left(\mathrm{~s}, 3 \mathrm{H}, \mathrm{CH}_{3} \mathrm{O}\right.$ ), 3.378 (dd, $\left.1 \mathrm{H}, J_{5^{\prime} a x, 4^{\prime}} 7.5, J_{5^{\prime} a x, 5^{\prime} e q}-12.0 \mathrm{~Hz}, \mathrm{H}_{-5} 5^{\prime} a x\right), 3.279\left(\mathrm{~m}, 1 \mathrm{H}, J_{5,6 \mathrm{a}} 4.8, J_{5,6 \mathrm{~b}} 9.9 \mathrm{~Hz}\right.$, $\mathrm{H}-5), 2.074,2.056$, and $2.047(3 \mathrm{~s}$, each $3 \mathrm{H}, 3 \mathrm{Ac}) ;{ }^{13} \mathrm{C}, \delta 169.7(2 \mathrm{C})$ and $169.2(3$ $\left.\mathrm{COCH}_{3}\right), 138.1,137.4,128.7-127.4$, and $125.9\left(C_{6} \mathrm{H}_{5} \mathrm{CH}_{2} \mathrm{O}\right.$ and $\left.C_{6} \mathrm{H}_{5} \mathrm{CH}\right), 102.3$, 101.3, and 100.6 (C-1,1' and $\mathrm{PhCH}), 78.0,76.0,74.9,70.3,70.0,68.8$, and 67.4 $\left(\mathrm{C}-2,3,4,5,2^{\prime}, 3^{\prime}, 4^{\prime}\right), 71.5,68.4$, and $61.4\left(\mathrm{C}-6,5^{\prime}\right.$ and $\left.\mathrm{PhCH}_{2} \mathrm{O}\right), 56.8\left(\mathrm{CH}_{3} \mathrm{O}\right), 20.7$ $\left(\mathrm{COCH}_{3}\right)$. Anal. Calcd for $\mathrm{C}_{32} \mathrm{H}_{38} \mathrm{O}_{13}: \mathrm{C}, 60.94 ; \mathrm{H}, 6.07$. Found: $\mathrm{C}, 60.43 ; \mathrm{H}, 6.00$. Methyl 3,6-di-O-benzyl-2-O-(2,3,4-tri-O-acetyl- $\beta$-D-xylopyranosyl)- $\beta$-D-mannopyranoside (10).-A solution of $9(627 \mathrm{mg}, 1.0 \mathrm{mmol})$ and sodium cyanoborohydride $(750 \mathrm{mg}, 12 \mathrm{mmol})$ in tetrahydrofuran $(18 \mathrm{~mL})$ containing $3 \mathrm{~A}$ molecular sieves $\left(2.0 \mathrm{~g}\right.$ ) was stirred for $30 \mathrm{~min}$ under $\mathrm{N}_{2}$. Then a saturated solution of $\mathrm{HCl}$ in diethyl ether was added until the evolution of gas ceased, and the mixture was stirred for $30 \mathrm{~min}$. TLC $\left(95: 5 \mathrm{CH}_{2} \mathrm{Cl}_{2}\right.$-acetone) then showed the conversion of 9 $\left(R_{f} 0.76\right)$ into $10\left(R_{f} 0.22\right)$. The mixture was diluted with $\mathrm{CH}_{2} \mathrm{Cl}_{2}(200 \mathrm{~mL})$, filtered through Celite, washed with water, aq $5 \% \mathrm{NaHCO}_{3}$, and water, dried $\left(\mathrm{MgSO}_{4}\right)$, filtered, and concentrated. Column chromatography $\left(95: 5 \mathrm{CH}_{2} \mathrm{Cl}_{2}-\right.$ acetone) of the residue gave 10, isolated as a white solid $(421 \mathrm{mg}, 67 \%) ;[\alpha]_{D}$ $-101^{\circ}$ (c 1). NMR data $\left(\mathrm{CDCl}_{3}\right):{ }^{1} \mathrm{H}, \delta$ 7.38-7.27 (m, $\left.10 \mathrm{H}, 2 \mathrm{Ph}\right), 5.175(\mathrm{t}, 1 \mathrm{H}$, $\left.J_{3^{\prime}, 4^{\prime}}, 8.0 \mathrm{~Hz}, \mathrm{H}-3^{\prime}\right), 4.996\left(\mathrm{dd}, 1 \mathrm{H}, J_{z^{\prime}, 3^{\prime}} 8.0 \mathrm{~Hz}, \mathrm{H}-2^{\prime}\right), 4.917\left(\mathrm{~m}, 1 \mathrm{H}, J_{4^{\prime}, S^{\prime} e q} 4.8\right.$, $\left.J_{4^{\prime}, 5^{\prime} a x} 8.0 \mathrm{~Hz}, \mathrm{H}-4^{\prime}\right), 4.902\left(\mathrm{~d}, 1 \mathrm{H}, J_{1^{\prime}, 2^{\prime}} 6.3 \mathrm{~Hz}, \mathrm{H}-1^{\prime}\right), 4.799$ and 4.495 (2 d, each 1 $\mathrm{H}, \mathrm{PhCH} \mathrm{H}_{2} \mathrm{O}$ ), 4.602 and 4.557 ( $2 \mathrm{~d}$, each $1 \mathrm{H}, \mathrm{PhCH}_{2} \mathrm{O}$ ), 4.267 (s, $\left.1 \mathrm{H}, \mathrm{H}-1\right), 4.228$ 
(dd, $\left.1 \mathrm{H}, J_{5^{\prime} e q, 5^{\prime} a x}-11.9 \mathrm{~Hz}, \mathrm{H}-5^{\prime} e q\right), 4.150$ (d, $\left.1 \mathrm{H}, J_{2,3} 2.9 \mathrm{~Hz}, \mathrm{H}-2\right), 3.821$ (dd, 1 $\left.\mathrm{H}, J_{6 \mathrm{a}, 5} 3.3, J_{6 \mathrm{a}, 6 \mathrm{~b}}-10.4 \mathrm{~Hz}, \mathrm{H}-6 \mathrm{a}\right), 3.743\left(\mathrm{~m}, 1 \mathrm{H}, J_{4,5} 9.4 \mathrm{~Hz}, \mathrm{H}-4\right), 3.658$ (dd, $1 \mathrm{H}$, $\left.J_{6 \mathrm{~b}, 5} 6.4 \mathrm{~Hz}, \mathrm{H}-6 \mathrm{~b}\right), 3.472\left(\mathrm{~s}, 3 \mathrm{H}, \mathrm{CH}_{3} \mathrm{O}\right), 3.401(\mathrm{~m}, 1 \mathrm{H}, \mathrm{H}-5), 3.356(\mathrm{dd}, 1 \mathrm{H}$, H-5'ax), 3.289 (dd, $1 \mathrm{H}, J_{3,4} 9.4 \mathrm{~Hz}, \mathrm{H}-3$ ), 2.527 (d, $1 \mathrm{H}, J_{\mathrm{HO}-4,4} 1.9 \mathrm{~Hz}, \mathrm{HO}-4$ ), 2.047, 2.011, and $1.989(3 \mathrm{~s}$, each $3 \mathrm{H}, 3 \mathrm{Ac}) ;{ }^{13} \mathrm{C}, \delta 169.9,169.7$, and $169.3(3$ $\left.\mathrm{COCH}_{3}\right), 138.0,137.5$, and 128.3-127.5 $\left(\mathrm{C}_{6} \mathrm{H}_{5} \mathrm{CH}_{2} \mathrm{O}\right), 101.5$ and $100.6\left(\mathrm{C}-1,1^{\prime}\right)$, 79.5, 75.1, 72.4, 70.0, 68.9, and $67.5(2 \mathrm{C})\left(\mathrm{C}-2,3,4,5,2^{\prime}, 3^{\prime}, 4^{\prime}\right), 73.3,70.6,70.4$, and 61.4 (C-6,5' and $\left.2 \mathrm{PhCH}_{2} \mathrm{O}\right), 56.6\left(\mathrm{CH}_{3} \mathrm{O}\right), 20.6\left(\mathrm{COCH}_{3}\right)$. Anal. Calcd for $\mathrm{C}_{32} \mathrm{H}_{40} \mathrm{O}_{13}: \mathrm{C}, 60.75 ; \mathrm{H}, 6.37$. Found: $\mathrm{C}, 60.52 ; \mathrm{H}, 6.42$.

Methyl 4-O-acetyl-3,6-di-O-benzyl-2-O-(2,3,4-tri-O-acetyl- $\beta$-D-xylopyranosyl)- $\beta$ D-mannopyranoside (11). - A solution of $10(336 \mathrm{mg}, 0.52 \mathrm{mmol})$ in $1: 1$ pyridineacetic anhydride $(30 \mathrm{~mL})$ was stirred for $16 \mathrm{~h}$ at room temperature, and toluene $(3 \times 15 \mathrm{~mL})$, EtOH $(3 \times 15 \mathrm{~mL})$, and $\mathrm{CH}_{2} \mathrm{Cl}_{2}(3 \times 15 \mathrm{~mL})$ were evaporated from the residue, to give 11 , isolated as a yellow solid $(350 \mathrm{mg}, 99 \%) ; R_{f} 0.60(95: 5$ $\mathrm{CH}_{2} \mathrm{Cl}_{2}$-acetone). NMR data $\left(\mathrm{CDCl}_{3}\right):{ }^{1} \mathrm{H}, \delta$ 7.35-7.16 (m, $\left.10 \mathrm{H}, 2 \mathrm{Ph}\right), 5.145(\mathrm{t}, 1$ $\left.\mathrm{H}, J_{4,3}=J_{4,5}=9.7 \mathrm{~Hz}, \mathrm{H}-4\right), 4.711$ and $4.492\left(2 \mathrm{~d}\right.$, each $\left.1 \mathrm{H}, \mathrm{PhCH} \mathrm{H}_{2} \mathrm{O}\right), 4.528(\mathrm{~s}, 2$ $\mathrm{H}, \mathrm{PhCH} \mathrm{H}_{2} \mathrm{O}$ ), 4.290 (s, $\left.1 \mathrm{H}, \mathrm{H}-1\right), 4.152$ (d, $\left.1 \mathrm{H}, J_{2,3} 3.0 \mathrm{~Hz}, \mathrm{H}-2\right), 3.480$ (s, $3 \mathrm{H}$, $\mathrm{CH}_{3} \mathrm{O}$ ), 2.101, 2.071, 2.050, and $1.946(4 \mathrm{~s}$, each $3 \mathrm{H}, 4 \mathrm{Ac}) ;{ }^{13} \mathrm{C}, \delta 137.6$ and 128.2-127.4 $\left(\mathrm{C}_{6} \mathrm{H}_{5} \mathrm{CH}_{2} \mathrm{O}\right), 101.4$ and $99.0\left(\mathrm{C}-1,1^{\prime}\right), 73.5,70.7,70.1$, and $59.6\left(\mathrm{C}-6,5^{\prime}\right.$ and $\left.2 \mathrm{PhCH}_{2} \mathrm{O}\right), 56.7\left(\mathrm{CH}_{3} \mathrm{O}\right), 20.6\left(\mathrm{COCH}_{3}\right)$. Anal. Calcd for $\mathrm{C}_{34} \mathrm{H}_{42} \mathrm{O}_{14}: \mathrm{C}$, $60.53 ; \mathrm{H}, 6.27$. Found: $\mathrm{C}, 60.29 ; \mathrm{H}, 6.45$.

Methyl 4-O-acetyl-2-O-(2,3,4-tri-O-acetyl- $\beta$-D-xylopyranosyl)- $\beta$-D-mannopyranoside (12). - To a solution of $11(375 \mathrm{mg}, 0.58 \mathrm{mmol})$ in $1: 1 \mathrm{EtOH}-\mathrm{EtOAc}(12 \mathrm{~mL})$ were added acetic acid $(0.3 \mathrm{~mL})$ and $10 \% \mathrm{Pd}-\mathrm{C}(250 \mathrm{mg})$. Hydrogenolysis was performed at atmospheric pressure for $1 \mathrm{~h}$, the mixture was filtered and concentrated, and toluene $(3 \times 10 \mathrm{~mL})$ was evaporated from the residue, to yield 12 , isolated as a white solid ( $249 \mathrm{mg}, 90 \%) ; R_{f} 0.76\left(9: 1 \mathrm{CH}_{2} \mathrm{Cl}_{2}-\mathrm{MeOH}\right)$. NMR data $\left(\mathrm{CDCl}_{3}\right):{ }^{1} \mathrm{H}, \delta 4.745$ (d, $\left.1 \mathrm{H}, J_{1^{\prime}, 2^{\prime}} 6.9 \mathrm{~Hz}, \mathrm{H}-1^{\prime}\right), 4.380$ (s, $\left.1 \mathrm{H}, \mathrm{H}-1\right), 4.136$ (dd, 1 $\left.\mathrm{H}, J_{5^{\prime} e q, 4^{\prime}} 5.1, J_{5^{\prime} e q, 5^{\prime} a x}-11.8 \mathrm{~Hz}, \mathrm{H}-5^{\prime} e q\right), 4.000\left(\mathrm{~d}, 1 \mathrm{H}, J_{2,3} 3.1 \mathrm{~Hz}, \mathrm{H}-2\right), 3.493$ (s, $3 \mathrm{H}, \mathrm{CH}_{3} \mathrm{O}$ ), 2.846 and 2.810 ( $2 \mathrm{bs}$, each $\left.1 \mathrm{H}, \mathrm{HO}-3,6\right), 2.104,2.060,2.058$, and $2.052(4 \mathrm{~s}$, each $3 \mathrm{H}, 4 \mathrm{Ac}) ;{ }^{13} \mathrm{C}, \delta 170.5$ and $169.8-169.6\left(\mathrm{COCH}_{3}\right), 101.1\left(\mathrm{C}-1,1^{\prime}\right)$, 77.8, 74.2, 71.1, 70.5 (2 C), 69.0, and 68.5 (C-2,3,4,5, $\left.2^{\prime}, 3^{\prime}, 4^{\prime}\right), 61.8$ and $61.3\left(\mathrm{C}-6,5^{\prime}\right)$, $56.9\left(\mathrm{CH}_{3} \mathrm{O}\right), 20.8,20.6$, and $20.5(2 \mathrm{C})\left(4 \mathrm{COCH}_{3}\right)$. Anal. Calcd for $\mathrm{C}_{20} \mathrm{H}_{30} \mathrm{O}_{14}$ : C, 48.58; H, 6.12. Found: C, 48.42; H, 6.33.

Benzyl 4,6-O-isopropylidene-3-O-methyl- $\beta$-D-glucopyranoside (13).-To a solution of benzyl 3-O-methyl- $\beta$-D-glucopyranoside ${ }^{16}(6.0 \mathrm{~g}, 21.1 \mathrm{mmol})$ in 2,2-dimethoxypropane $(50 \mathrm{~mL})$ was added $p$-toluenesulfonic acid monohydrate $(100 \mathrm{mg})$, and the mixture was stirred for $45 \mathrm{~min}$. TLC (95:5 $\mathrm{CH}_{2} \mathrm{Cl}_{2}$-acetone) then showed the reaction to be complete, and solid $\mathrm{NaHCO}_{3}$ was added. The mixture was diluted with $\mathrm{CH}_{2} \mathrm{Cl}_{2}(300 \mathrm{~mL})$, washed with water, dried $\left(\mathrm{MgSO}_{4}\right)$, filtered, and concentrated. The residue was crystallised from EtOH to give $13(6.0 \mathrm{~g}, 88 \%)$; $[\alpha]_{\mathrm{D}}$ $-69^{\circ}$ ( c 1); mp $108^{\circ} \mathrm{C} ; R_{f} 0.43$ (95:5 $\mathrm{CH}_{2} \mathrm{Cl}_{2}$-acetone). NMR data $\left(\mathrm{CDCl}_{3}\right):{ }^{1} \mathrm{H}$, $\delta$ 7.37-7.31 (m, $5 \mathrm{H}, \mathrm{Ph}), 4.911$ and $4.632\left(2 \mathrm{~d}\right.$, each $\left.1 \mathrm{H}, \mathrm{PhCH} \mathrm{H}_{2} \mathrm{O}\right), 4.448(\mathrm{~d}, 1 \mathrm{H}$, 
$\left.J_{1,2} 7.7 \mathrm{~Hz}, \mathrm{H}-1\right), 3.944\left(\mathrm{dd}, 1 \mathrm{H}, J_{6 \mathrm{a}, 5} 5.4, J_{6 \mathrm{a}, 6 \mathrm{~b}}-10.5 \mathrm{~Hz}, \mathrm{H}-6 \mathrm{a}\right), 3.811\left(\mathrm{t}, 1 \mathrm{H}, J_{6 \mathrm{~b}, 5}\right.$ $10.5 \mathrm{~Hz}, \mathrm{H}-6 \mathrm{~b}), 3.661\left(\mathrm{t}, 1 \mathrm{H}, J_{4,3}=J_{4,5}=9.4 \mathrm{~Hz}, \mathrm{H}-4\right), 3.598\left(\mathrm{~s}, 3 \mathrm{H}, \mathrm{CH}_{3} \mathrm{O}\right), 3.505$ $\left(\mathrm{m}, 1 \mathrm{H}, J_{2,3} 8.9 \mathrm{~Hz}, \mathrm{H}-2\right), 2.399\left(\mathrm{~d}, 1 \mathrm{H}, J_{\mathrm{HO}-2,2} 2.2 \mathrm{~Hz}, \mathrm{HO}-2\right), 1.512$ and $1.425(2$ $\mathrm{s}$, each $\left.3 \mathrm{H}, \mathrm{CMe}_{2}\right) ;{ }^{13} \mathrm{C}, \delta 137.0$ and $128.3-127.8\left(C_{6} \mathrm{H}_{5} \mathrm{CH}_{2} \mathrm{O}\right), 102.0$ and 99.1 (C-1 and $\mathrm{Me}_{2} \mathrm{C}$ ), 82.3, 73.9, 73.7, and 67.0 (C-2,3,4,5), 71.0 and 62.0 (C-6 and

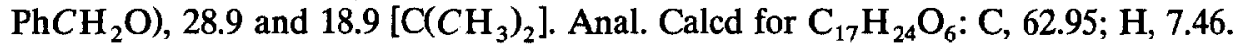
Found: C, 62.61; $\mathrm{H}, 7.45$.

Benzyl 4,6-O-isopropylidene-3-O-methyl- $\beta$-D-mannopyranoside (15).-A solution of $13(5.8 \mathrm{~g}, 17.9 \mathrm{mmol})$ in $1: 2$ acetic anhydride- $\mathrm{Me}_{2} \mathrm{SO}(100 \mathrm{~mL})$ was stirred for $20 \mathrm{~h}$. TLC (95:5 $\mathrm{CH}_{2} \mathrm{Cl}_{2}$-acetone) then showed the disappearance of $13\left(R_{f}\right.$ $0.43)$, and the formation of ulose $14\left(R_{f} 0.28-0.43\right)$ and a faster moving product $\left(R_{f} 0.66\right)$. After partial evaporation of the solvent using toluene $(3 \times 30 \mathrm{~mL})$, the residue was further concentrated in vacuo (bath $<60^{\circ} \mathrm{C}$ ). Column chromatography (95:5 $\mathrm{CH}_{2} \mathrm{Cl}_{2}$-acetone) of the residue gave 14 , isolated as a syrup (4.6 $\mathrm{g}, 79 \%$ ); $[\alpha]_{\mathrm{D}}+20^{\circ}(c 1)$ for $\mathrm{C}-2_{\mathrm{CO}}: \mathrm{C}-2_{\mathrm{C(OH})_{2}}=0.2: 1 .{ }^{13} \mathrm{C} \mathrm{NMR}$ data $\left(\mathrm{CDCl}_{3}\right): \delta 187.7$ (C-2 as carbonyl) and 93.6 (C-2 as gem-diol).

To a solution of $14(3.0 \mathrm{~g}, 9.2 \mathrm{mmol})$ in $1: 1 \mathrm{CH}_{2} \mathrm{Cl}_{2}-\mathrm{MeOH}(50 \mathrm{~mL})$ containing Kieselgel 60 (70-230 mesh, $1.0 \mathrm{~g})$ was added $\mathrm{NaBH}_{4}(1.4 \mathrm{~g}, 37 \mathrm{mmol})$, as described for 7. After $30 \mathrm{~min}$, TLC ( $95: 5 \mathrm{CH}_{2} \mathrm{Cl}_{2}$-acetone) showed the formation of $\mathbf{1 3}\left(R_{f}\right.$ $0.43)$ and $15\left(R_{f} 0.17\right)$ in the ratio $\sim 1: 10$. The mixture was diluted with $\mathrm{CH}_{2} \mathrm{Cl}_{2}$ $(300 \mathrm{~mL})$, filtered, washed with water, dried $\left(\mathrm{MgSO}_{4}\right)$, filtered, and concentrated. Column chromatography $\left[3: 2\right.$ light petroleum $\left(\mathrm{bp} 40-60^{\circ} \mathrm{C}\right)$-EtOAc] of the residue first gave $13(200 \mathrm{mg}, 7 \%)$, and then $15(2.4 \mathrm{~g}, 79 \%)$, both isolated as a white solid. Compound 15: $[\alpha]_{\mathrm{D}}-95^{\circ}(c 1) ; R_{f}$ 0.17. NMR data $\left(\mathrm{CDCl}_{3}\right):{ }^{1} \mathrm{H}, \delta 7.41-7.29(\mathrm{~m}$, $5 \mathrm{H}, \mathrm{Ph}), 4.934$ and $4.664\left(2 \mathrm{~d}\right.$, each $\left.1 \mathrm{H}, \mathrm{PhCH} \mathrm{H}_{2} \mathrm{O}\right), 4.523(\mathrm{~s}, 1 \mathrm{H}, \mathrm{H}-1), 4.176(\mathrm{bd}$, $\left.1 \mathrm{H}, J_{2,3} 3.2 \mathrm{~Hz}, \mathrm{H}-2\right), 4.084(\mathrm{t}, 1 \mathrm{H}, \mathrm{H}-4), 3.492\left(\mathrm{~s}, 3 \mathrm{H}, \mathrm{CH}_{3} \mathrm{O}\right.$ ), 3.278 (dd, $1 \mathrm{H}, J_{3,4}$ $9.3 \mathrm{~Hz}, \mathrm{H}-3), 3.180\left(\mathrm{~m}, 1 \mathrm{H}, J_{5,6 \mathrm{a}} 5.8, J_{5,6 \mathrm{~b}}=J_{5,4}=9.8 \mathrm{~Hz}, \mathrm{H}-5\right), 2.482(\mathrm{bs}, 1 \mathrm{H}$, $\mathrm{HO}-2), 1.527$ and $1.431\left(2 \mathrm{~s}\right.$, each $\left.3 \mathrm{H}, \mathrm{CMe}_{2}\right) ;{ }^{13} \mathrm{C}, \delta 136.3$ and $128.2-127.8$ $\left(C_{6} \mathrm{H}_{5} \mathrm{CH}_{2} \mathrm{O}\right.$ ), 99.5 and $98.4\left(\mathrm{C}-1\right.$ and $\left.\mathrm{Me}_{2} C\right)$, 79.4, 70.1, 68.2, and $67.6(\mathrm{C}-2,3,4,5)$, 70.4 and $61.8\left(\mathrm{C}-6\right.$ and $\left.\mathrm{PhCH}_{2} \mathrm{O}\right), 57.3\left(\mathrm{CH}_{3} \mathrm{O}\right), 28.9$ and 18.9 [ $\left.\mathrm{C}\left(\mathrm{CH}_{3}\right)_{2}\right]$. Anal. Calcd for $\mathrm{C}_{17} \mathrm{H}_{24} \mathrm{O}_{6}: \mathrm{C}, 62.95 ; \mathrm{H}, 7.46$. Found: $\mathrm{C}, 63.33 ; \mathrm{H}, 7.31$.

Benzyl 2,4,6-tri-O-acetyl-3-O-methyl- $\beta$-D-mannopyranoside (16). - A solution of $15(2.2 \mathrm{~g}, 6.8 \mathrm{mmol})$ in $1: 1$ acetic acid-water $(20 \mathrm{~mL})$ was boiled under reflux for $45 \mathrm{~min}$, then concentrated, and toluene $(3 \times 20 \mathrm{~mL})$ was evaporated from the residue. The residue was dissolved in 1:1 pyridine-acetic anhydride $(20 \mathrm{~mL})$, stirred for $16 \mathrm{~h}$ at room temperature, and concentrated, and toluene $(3 \times 10 \mathrm{~mL})$, EtOH $(3 \times 10 \mathrm{~mL})$, and $\mathrm{CH}_{2} \mathrm{Cl}_{2}(3 \times 10 \mathrm{~mL})$ were evaporated from the residue to yield 16, isolated as a yellow syrup $(2.6 \mathrm{~g}, 93 \%) ; R_{f} 0.43\left(95: 5 \mathrm{CH}_{2} \mathrm{Cl}_{2}\right.$-acetone). NMR data $\left(\mathrm{CDCl}_{3}\right):{ }^{1} \mathrm{H}, \delta 7.39-7.32(\mathrm{~m}, 5 \mathrm{H}, \mathrm{Ph}), 5.567\left(\mathrm{bd}, 1 \mathrm{H}, J_{2,3} 3.3 \mathrm{~Hz}\right.$, $\mathrm{H}-2), 5.132\left(\mathrm{t}, 1 \mathrm{H}, J_{4,5} 9.8 \mathrm{~Hz}, \mathrm{H}-4\right), 4.893$ and $4.665\left(2 \mathrm{~d}\right.$, each $1 \mathrm{H}, \mathrm{PhCH} \mathrm{H}_{2} \mathrm{O}$ ), 4.518 (d, $\left.1 \mathrm{H}, J_{1,2} 0.9 \mathrm{~Hz}, \mathrm{H}-1\right), 4.293$ (dd, $\left.1 \mathrm{H}, J_{6 \mathrm{~b}, 5} 5.9 \mathrm{~Hz}, \mathrm{H}-6 \mathrm{~b}\right), 4.183$ (dd, $1 \mathrm{H}$, $\left.J_{6 \mathrm{a}, 5} 2.7, J_{6 \mathrm{a}, 6 \mathrm{~b}}-12.2 \mathrm{~Hz}, \mathrm{H}-6 \mathrm{a}\right), 3.541(\mathrm{~m}, 1 \mathrm{H}, \mathrm{H}-5), 3.335\left(\mathrm{~s}, 3 \mathrm{H}, \mathrm{CH}_{3} \mathrm{O}\right), 3.321$ (dd, $1 \mathrm{H}, J_{3,4} 9.8 \mathrm{~Hz}, \mathrm{H}-3$ ), 2.190, 2.114, and $2.072\left(3 \mathrm{~s}\right.$, each $3 \mathrm{H}, 3 \mathrm{Ac}$ ); ${ }^{13} \mathrm{C}, \delta$ 
$170.5,170.2$, and $169.5\left(3 \mathrm{COCH}_{3}\right), 136.3,128.2$, and $127.8\left(C_{6} \mathrm{H}_{5} \mathrm{CH}_{2} \mathrm{O}\right), 96.7$ (C-1), 79.3, 72.2, 67.4, and 66.8 (C-2,3,4,5), 70.1 and $62.6\left(\mathrm{C}-6\right.$ and $\left.\mathrm{PhCH}_{2} \mathrm{O}\right), 57.4$ $\left(\mathrm{CH}_{3} \mathrm{O}\right), 20.7$ and $20.6(2 \mathrm{C})\left(3 \mathrm{COCH}_{3}\right)$. Anal. Calcd for $\mathrm{C}_{20} \mathrm{H}_{26} \mathrm{O}_{9}: \mathrm{C}, 58.52 ; \mathrm{H}$, 6.39. Found: $\mathrm{C}, 58.24 ; \mathrm{H}, 6.21$.

2,4,6-Tri-O-acetyl-3-O-methyl- $\alpha$-D-mannopyranosyl trichloroacetimidate (18).To a solution of $16(2.5 \mathrm{~g}, 6.1 \mathrm{mmol})$ in $1: 1 \mathrm{EtOH}-\mathrm{EtOAc}(30 \mathrm{~mL})$ were added acetic acid $(0.5 \mathrm{~mL})$ and $10 \% \mathrm{Pd}-\mathrm{C}(250 \mathrm{mg})$. Hydrogenolysis, and work-up as described for 6, gave 17, isolated as a syrup $(1.9 \mathrm{~g}, 97 \%) ; R_{f} 0.16\left(95: 5 \mathrm{CH}_{2} \mathrm{Cl}_{2}-\right.$ acetone). Anal. Calcd for $\mathrm{C}_{13} \mathrm{H}_{20} \mathrm{O}_{9}: \mathrm{C}, 48.75 ; \mathrm{H}, 6.29$. Found: $\mathrm{C}, 48.12 ; \mathrm{H}, 6.23$.

To a solution of $17(1.9 \mathrm{~g}, 5.9 \mathrm{mmol})$ in $\mathrm{CH}_{2} \mathrm{Cl}_{2}(25 \mathrm{~mL})$ and trichloroacetonitrile $(7.3 \mathrm{~mL}, 73 \mathrm{mmol})$ was added 1,8-diazabicyclo[5.4.0]undec-7-ene $(1.0 \mathrm{~mL}, 6.7$ $\mathrm{mmol}$ ), and the mixture was stirred for $1 \mathrm{~h}$. Then the reaction was complete (TLC 95:5 $\mathrm{CH}_{2} \mathrm{Cl}_{2}$-acetone, $R_{f} \quad 0.52$ ), and the mixture was concentrated. Column chromatography (95:5 $\mathrm{CH}_{2} \mathrm{Cl}_{2}$-acetone) of the residue gave 18 , isolated as a yellow syrup $(2.0 \mathrm{~g}, 73 \%) ;[\alpha]_{\mathrm{D}}+23^{\circ}(c)$ ). NMR data $\left(\mathrm{CDCl}_{3}\right):{ }^{1} \mathrm{H}, \delta 8.799(\mathrm{~s}, 1$ $\mathrm{H}, \mathrm{NH}), 6.303$ (d, $\left.1 \mathrm{H}, J_{1,2} 2.0 \mathrm{~Hz}, \mathrm{H}-1\right), 5.536$ (dd, $\left.1 \mathrm{H}, J_{2,3} 3.4 \mathrm{~Hz}, \mathrm{H}-2\right), 5.293$ (t, $1 \mathrm{H}, J_{4,5} 10.0 \mathrm{~Hz}, \mathrm{H}-4$ ), 4.247 (dd, $1 \mathrm{H}, J_{6 \mathrm{~b}, 5} 5.4, J_{6 \mathrm{~b}, 6 \mathrm{a}}-12.5 \mathrm{~Hz}, \mathrm{H}-6 \mathrm{~b}$ ), 3.732 (dd, $\left.1 \mathrm{H}, J_{3,4} 10.0 \mathrm{~Hz}, \mathrm{H}-3\right), 3.395\left(\mathrm{~s}, 3 \mathrm{H}, \mathrm{CH}_{3} \mathrm{O}\right), 2.204,2.119$, and 2.083 ( $3 \mathrm{~s}$, each 3 $\mathrm{H}, 3 \mathrm{Ac}) ;{ }^{13} \mathrm{C}, \delta 170.0,169.3$, and $169.2\left(3 \mathrm{COCH}_{3}\right), 159.0\left(\mathrm{OCNHCCl}_{3}\right), 94.3$ (C-1), 90.0 ( $\left.\mathrm{OCNHCCl}_{3}\right), 76.4,70.8,66.4$, and 65.7 (C-2,3,4,5), 61.8 (C-6), 57.5 $\left(\mathrm{CH}_{3} \mathrm{O}\right)$, $20.3\left(\mathrm{COCH}_{3}\right)$. Anal. Caled for $\mathrm{C}_{15} \mathrm{H}_{20} \mathrm{O}_{9} \mathrm{NCl}_{3}$ : C, 38.77; H, 4.34. Found: C, 38.40; $\mathrm{H}, 4.45$.

Methyl 4-O-acetyl-3,6-di-O-(2,4,6-tri-O-acetyl-3-O-methyl- $\alpha$-D-mannopyranosyl)2-O-(2,3,4-tri-O-acetyl- $\beta$-D-xylopyranosyl)- $\beta$-D-mannopyranoside (19).-A solution of $12(50 \mathrm{mg}, 0.1 \mathrm{mmol})$ and $18(170 \mathrm{mg}, 0.37 \mathrm{mmol})$ in $\mathrm{CH}_{2} \mathrm{Cl}_{2}(10 \mathrm{~mL})$ containing powdered 4A molecular sieves $(1.0 \mathrm{~g})$ was stirred for $30 \mathrm{~min}$ under $\mathrm{N}_{2}$. A solution of trimethylsilyl triflate $(40 \mu \mathrm{L})$ in $\mathrm{CH}_{2} \mathrm{Cl}_{2}(2 \mathrm{~mL})$ was added dropwise at $-40^{\circ} \mathrm{C}$, and after $1 \mathrm{~h}$, when TLC showed the disappearance of 12 and a new compound 19 ( $R_{f} 0.18,9: 1 \mathrm{CH}_{2} \mathrm{Cl}_{2}$-acetone), pyridine $(1 \mathrm{~mL}$ ) was added, the mixture was filtered through Celite and concentrated, and toluene $(3 \times 10 \mathrm{~mL})$ was evaporated from the rcsidue. Column chromatography $\left(9: 1 \mathrm{CH}_{2} \mathrm{Cl}_{2}\right.$-acetone) of the residue gave 19, isolated as a white solid $(72 \mathrm{mg}, 71 \%) ;[\alpha]_{\mathrm{D}}-34^{\circ}(c 1)$. NMR data $\left(\mathrm{CDCl}_{3}\right):{ }^{1} \mathrm{H}, \delta 5.438$ and $5.102\left(2 \mathrm{dd}\right.$, each $1 \mathrm{H}, J_{2^{\prime \prime}, 1^{\prime \prime}}=J_{2^{m \prime \prime}, 1^{\prime \prime \prime}}=1.7, J_{2^{\prime \prime}, 3^{\prime \prime}}=J_{2^{m}, 3^{\prime \prime \prime}}$ $=3.4, \mathrm{H}-2^{\prime \prime}, 2^{\prime \prime \prime}$ ), 5.028 (dd, $1 \mathrm{H}, J_{2^{\prime}, 3^{\prime}} 9.1 \mathrm{~Hz}, \mathrm{H}-2^{\prime}$ ), 4.908 and 4.814 (bs and d, each $\left.1 \mathrm{H}, \mathrm{H}-1^{\prime \prime}, 1^{\prime \prime \prime}\right), 4.900\left(\mathrm{~m}, 1 \mathrm{H}, \mathrm{H}-4^{\prime}\right), 4.765\left(\mathrm{~d}, 1 \mathrm{H}, J_{1^{\prime}, 2^{\prime}} 7.1 \mathrm{~Hz}, \mathrm{H}-1^{\prime}\right), 4.353$ (s, $1 \mathrm{H}, \mathrm{H}-1$ ), 4.275 (dd, $\left.1 \mathrm{H}, J_{5^{\prime} e q, 4^{\prime}} 5.6, J_{5^{\prime} e q, 5^{\prime} a x}-12.3 \mathrm{~Hz}, \mathrm{H}-5^{\prime} e q\right), 3.497$ and 3.398 $\left(2 \mathrm{~s}, 3\right.$ and $6 \mathrm{H}, 3 \mathrm{CH}_{3} \mathrm{O}$ ), $3.306\left(\mathrm{dd}, 1 \mathrm{H}, J_{5^{\prime} a x, 4^{\prime}} 9.0 \mathrm{~Hz}, \mathrm{H}-5^{\prime} a x\right), 2.148,2.144$, 2.128, 2.098, 2.089, 2.077, 2.068, and $2.043(8 \mathrm{~s}, 3,3,3,3,6,6,3,3 \mathrm{H}, 10 \mathrm{Ac}) ;{ }^{13} \mathrm{C}, \delta$ 170.5-169.0 $\left(\mathrm{COCH}_{3}\right), 101.3(2 \mathrm{C}), 99.9$, and $97.6\left(\mathrm{C}-1,1^{\prime}, 1^{\prime \prime}, 1^{\prime \prime \prime}\right), 78.5,76.4,76.2$, $75.9,72.6,70.8,70.3,69.4,68.7,68.5,68.3,68.0,67.8,67.5$, and 67.4 (C$\left.2,3,4,5,2^{\prime}, 3^{\prime}, 4^{\prime}, 2^{\prime \prime}, 3^{\prime \prime}, 4^{\prime \prime}, 5^{\prime \prime}, 2^{\prime \prime \prime}, 3^{\prime \prime \prime}, 4^{\prime \prime \prime}, 5^{\prime \prime \prime}\right), 66.4,63.0,62.5$, and $61.6\left(\mathrm{C}-6,5^{\prime}, 6^{\prime \prime}, 6^{\prime \prime \prime}\right)$, 58.0, 57.6, and $56.7\left(3 \mathrm{CH}_{3} \mathrm{O}\right)$, 20.8-20.5 $\left(\mathrm{COCH}_{3}\right)$. Anal. Calcd for $\mathrm{C}_{46} \mathrm{H}_{66} \mathrm{O}_{30}: \mathrm{C}$, 50.27 ; H, 6.05. Found: C, 50.15; H, 6.12 . 
Methyl 3,6-di-O-(3-O-methyl- $\alpha$-D-mannopyranosyl)-2-O- $\beta$-D-xylopyranosyl- $\beta$-Dmannopyranoside (2). - To a solution of $19(25 \mathrm{mg}, 24 \mu \mathrm{mol})$ in $\mathrm{MeOH}(5 \mathrm{~mL})$ was added $\mathrm{NaOMe}(\mathrm{pH} 10)$. After $16 \mathrm{~h}$, the solution was neutralised with Dowex-50 $\left(\mathrm{H}^{+}\right)$resin, filtered, and concentrated, and $\mathrm{CH}_{2} \mathrm{Cl}_{2}(3 \times 5 \mathrm{~mL})$ was evaporated from the residue, to give 2 , isolated as a white powder $(13 \mathrm{mg}, 90 \%) ;[\alpha]_{\mathrm{D}}+0.4^{\circ}(c$ $\left.0.5, \mathrm{H}_{2} \mathrm{O}\right) ; R_{f} 0.50$ (2:1:1 1-butanol-MeOH-water). ${ }^{13} \mathrm{C}$ NMR data $\left(\mathrm{D}_{2} \mathrm{O}\right): \delta$ $106.4,103.3,102.6$, and $100.9\left(\mathrm{C}-1,1^{\prime}, 1^{\prime \prime}, 1^{\prime \prime \prime}\right), 58.2,57.6$, and $57.5\left(3 \mathrm{CH}_{3} \mathrm{O}\right)$. For ${ }^{1} \mathrm{H}$ NMR data, see Table I.

Methyl 3-O-benzyl-4,6-O-prop-2-enylidene- $\beta$-D-glucopyranoside (20).-To a solution of methyl 3-O-benzyl- $\beta$-D-glucopyranoside ${ }^{11}(11.1 \mathrm{~g}, 39 \mathrm{mmol})$ in $N, N$-dimethylformamide $(33 \mathrm{~mL})$ and acrolein dimethyl acetal $(15.9 \mathrm{~mL}, 134 \mathrm{mmol})$ was added $p$-toluenesulfonic acid monohydrate $(250 \mathrm{mg})$, and the solution was stirred for $16 \mathrm{~h}$ at $60^{\circ} \mathrm{C}$. TLC (95:5 $\mathrm{CH}_{2} \mathrm{Cl}_{2}$-acetone) then showed the reaction to be complete, and triethylamine $(2 \mathrm{~mL}$ ) was added. The mixture was concentrated in vacuo (bath $\left.<60^{\circ} \mathrm{C}\right)$, diluted with $\mathrm{CH}_{2} \mathrm{Cl}_{2}(250 \mathrm{~mL})$, washed with water, dried $\left(\mathrm{MgSO}_{4}\right)$, filtered, and concentrated. Crystallisation of the residue from EtOH gave $20(9.4 \mathrm{~g}$, 75\%); mp $146^{\circ} \mathrm{C} ;[\alpha]_{\mathrm{D}}-6^{\circ}(c)$ ); $R_{f} 0.51$ (95:5 $\mathrm{CH}_{2} \mathrm{Cl}_{2}$-acetone). NMR data $\left(\mathrm{CDCl}_{3}\right):{ }^{1} \mathrm{H}, \delta$ 7.40-7.28 (m, $\left.5 \mathrm{H}, \mathrm{Ph}\right), 5.879\left(\mathrm{~m}, 1 \mathrm{H}, \mathrm{H}_{2} \mathrm{C}=\mathrm{CHCH}\right), 5.509$ and $5.345\left(2 \mathrm{~m}\right.$, each $\left.1 \mathrm{H}, \mathrm{H}_{2} \mathrm{C}=\mathrm{CHCH}\right), 5.012\left(\mathrm{~m}, 1 \mathrm{H}, \mathrm{H}_{2} \mathrm{C}=\mathrm{CHCH}\right), 4.948$ and 4.771 ( $2 \mathrm{~d}$, each $1 \mathrm{H}, \mathrm{PhCH} \mathrm{H}_{2} \mathrm{O}$ ), 4.287 (d, $1 \mathrm{H}, J_{1,2} 7.6 \mathrm{~Hz}, \mathrm{H}-1$ ), 4.248 (dd, $1 \mathrm{H}, J_{6 \mathrm{a}, 5} 5.0$, $\left.J_{6 \mathrm{a}, 6 \mathrm{~b}}-10.5 \mathrm{~Hz}, \mathrm{H}-6 \mathrm{a}\right), 3.550\left(\mathrm{~s}, 3 \mathrm{H}, \mathrm{CH}_{3} \mathrm{O}\right), 3.351\left(\mathrm{~m}, 1 \mathrm{H}, J_{5,4}=J_{5,6 \mathrm{~b}}=9.5 \mathrm{~Hz}\right.$, $\mathrm{H}-5), 2.386\left(\mathrm{~d}, 1 \mathrm{H}, J_{\mathrm{HO}-2,2} 2.1 \mathrm{~Hz}, \mathrm{HO}-2\right) ;{ }^{13} \mathrm{C}, \delta 138.2$ and $128.3-127.7$ $\left(\mathrm{C}_{6} \mathrm{H}_{5} \mathrm{CH}_{2} \mathrm{O}\right), 133.4\left(\mathrm{H}_{2} \mathrm{C}=\mathrm{CHCH}\right), 118.9\left(\mathrm{H}_{2} \mathrm{C}=\mathrm{CHCH}\right), 104.0\left(\mathrm{H}_{2} \mathrm{C}=\mathrm{CHCH}\right)$, 100.3 (C-1), 80.9, 80.0, 74.0, and 66.1 (C-2,3,4,5), 74.4 and 68.2 (C-6 and $\mathrm{PhCH}_{2} \mathrm{O}$ ), 57.2 $\left(\mathrm{CH}_{3} \mathrm{O}\right)$. Anal. Calcd for $\mathrm{C}_{17} \mathrm{H}_{22} \mathrm{O}_{6}: \mathrm{C}, 63.34 ; \mathrm{H}, 6.88$. Found: $\mathrm{C}, 63.31 ; \mathrm{H}$, 6.76.

Methyl 3-O-benzyl-4,6-O-prop-2-enylidene- $\beta$-D-mannopyranoside (22). -A solution of $20(7.2 \mathrm{~g}, 22 \mathrm{mmol})$ in $1: 2$ acetic anhydride- $\mathrm{Me}_{2} \mathrm{SO}(10 \mathrm{~mL})$ was stirred for $20 \mathrm{~h}$. TLC $\left(95: 5 \mathrm{CH}_{2} \mathrm{Cl}_{2}\right.$-acetone) then showed the formation of the ulose $21\left(R_{f}\right.$ $0.23-0.35)$ and a faster moving product $\left(R_{f} 0.87\right)$. Work-up as described for 6 gave 21, isolated as a yellow solid (8.3 g). A purified sample gave $[\alpha]_{D}-50^{\circ}(c 1)$ for $\mathrm{C}-2_{\mathrm{CO}}: \mathrm{C}-2_{\mathrm{C}(\mathrm{OH})_{2}}=1: 1 .{ }^{13} \mathrm{C}$ NMR data $\left(\mathrm{CDCl}_{3}\right): \delta 187.9$ (C-2 as carbonyl) and 93.4 (C-2 as gem-diol).

To a solution of $21(7.1 \mathrm{~g}, 22 \mathrm{mmol})$ in $1: 1 \mathrm{CH}_{2} \mathrm{Cl}_{2}-\mathrm{MeOH}(200 \mathrm{~mL})$ containing Kieselgel $60(70-230$ mesh, $3.0 \mathrm{~g})$ was added $\mathrm{NaBH}_{4}(2.6 \mathrm{~g}, 68 \mathrm{mmol})$, as described for 7. After $30 \mathrm{~min}$, TLC $\left(95: 5 \mathrm{CH}_{2} \mathrm{Cl}_{2}\right.$-acetone) showed $20\left(R_{f} 0.51\right)$ and $22\left(R_{f}\right.$ $0.31)$ in the ratio $\sim 1: 6$. The mixture was diluted with $\mathrm{CH}_{2} \mathrm{Cl}_{2}(300 \mathrm{~mL})$, filtered, washed with water, dried $\left(\mathrm{MgSO}_{4}\right)$, filtered, and concentrated. Column chromatography [3:2 light petroleum (bp $40-60^{\circ} \mathrm{C}$ )-EtOAc] of the residue first gave 20 (800 $\mathrm{mg}, 11 \%)$, and then $22(4.0 \mathrm{~g}, 56 \%)$, both isolated as a white solid (yields calculated from 20). Compound 22: $[\alpha]_{\mathrm{D}}-16^{\wedge}\left(\begin{array}{ll}c & 1\end{array}\right) ; R_{f}$ 0.18. NMR data $\left(\mathrm{CDCl}_{3}\right):{ }^{1} \mathrm{H}, \delta$ 7.41-7.31 (m, $5 \mathrm{H}, \mathrm{Ph}), 5.889\left(\mathrm{~m}, 1 \mathrm{H}, \mathrm{H}_{2} \mathrm{C}=\mathrm{CHCH}\right), 5.518$ and $5.344(2 \mathrm{~m}$, each 1 $\left.\mathrm{H}, \mathrm{H}_{2} \mathrm{C}=\mathrm{CHCH}\right), 5.050\left(\mathrm{~m}, 1 \mathrm{H}, \mathrm{H}_{2} \mathrm{C}=\mathrm{CHCH}\right), 4.851$ and $4.746(2 \mathrm{~d}$, each $1 \mathrm{H}$, 
$\mathrm{PhCH}_{2} \mathrm{O}$ ), 4.386 (d, $\left.1 \mathrm{H}, J_{1,2} 1.2 \mathrm{~Hz}, \mathrm{H}-1\right), 4.229$ (dd, $1 \mathrm{H}, J_{6 \mathrm{a}, 5} 4.9, J_{6 \mathrm{a}, 6 \mathrm{~b}}-10.5$ Hz, H-6a), 4.090 (m, $1 \mathrm{H}, \mathrm{H}-2$ ), 3.973 (t, $\left.1 \mathrm{H}, J_{4,5} 9.5 \mathrm{~Hz}, \mathrm{H}-4\right), 3.739$ (t, $1 \mathrm{H}, J_{6 \mathrm{~b}, 5}$ $10.4 \mathrm{~Hz}, \mathrm{H}-6 \mathrm{~b}$ ), 3.592 (dd, $1 \mathrm{H}, J_{2,3} 3.3, J_{3,4} 9.5 \mathrm{~Hz}, \mathrm{H}-3$ ), $3.540\left(\mathrm{~s}, 3 \mathrm{H}, \mathrm{CH}_{3} \mathrm{O}\right.$ ), $3.251(\mathrm{~m}, 1 \mathrm{H}, \mathrm{H}-5), 2.488\left(\mathrm{~d}, 1 \mathrm{H}, J_{\mathrm{HO}-2,2} 1.5 \mathrm{~Hz}, \mathrm{HO}-2\right) ;{ }^{13} \mathrm{C}, \delta 133.6$ $\left(\mathrm{H}_{2} \mathrm{C}=\mathrm{CHCH}\right), 128.4$ and $127.8\left(C_{6} \mathrm{H}_{5} \mathrm{CH}_{2} \mathrm{O}\right), 118.9\left(\mathrm{H}_{2} \mathrm{C}=\mathrm{CHCH}\right), 101.3$ and 100.6 (C-1 and $\left.\mathrm{H}_{2} \mathrm{C}=\mathrm{CHCH}\right), 77.0,76.6,69.6$, and $66.7(\mathrm{C}-2,3,4,5), 72.4$ and 68.1 (C-6 and $\mathrm{PhCH}_{2} \mathrm{O}$ ), $57.2\left(\mathrm{CH}_{3} \mathrm{O}\right)$. Anal. Calcd for $\mathrm{C}_{17} \mathrm{H}_{22} \mathrm{O}_{6}: \mathrm{C}, 63.34 ; \mathrm{H}, 6.88$. Found: $\mathrm{C}, 63.42 ; \mathrm{H}, 6.84$.

Methyl 3-O-benzyl-4,6-O-prop-2-enylidene-2-O-(2,3,4-tri-O-acetyl- $\beta$-D-xylopyranosyl)- $\beta$-D-mannopyranoside (23). -A solution of $22(2.1 \mathrm{~g}, 6.5 \mathrm{mmol})$ and 2,3,4-tri- $O$-acetyl- $\alpha$-D-xylopyranosyl trichloroacetimidate ${ }^{14}(8 ; 3.6 \mathrm{~g}, 8.6 \mathrm{mmol})$ in $\mathrm{CH}_{2} \mathrm{Cl}_{2}(50 \mathrm{~mL})$ containing powdered $4 \mathrm{~A}$ molecular sieves $(5.0 \mathrm{~g})$ was stirred for 30 min under $\mathrm{N}_{2}$. A solution of trimethylsilyl triflate $(300 \mu \mathrm{L})$ in $\mathrm{CH}_{2} \mathrm{Cl}_{2}(10 \mathrm{~mL})$ was added dropwise at $-40^{\circ} \mathrm{C}$. After $75 \mathrm{~min}$, when TLC showed the disappearance of 22 and a new compound $23\left(R_{f} 0.45,95: 5 \mathrm{CH}_{2} \mathrm{Cl}_{2}\right.$-acetone), pyridine $(1 \mathrm{~mL})$ was added, the mixture was filtered through Celite and concentrated, and toluene $(3 \times 10 \mathrm{~mL})$ was evaporated from the residue. Column chromatography [1:1 light petroleum (bp 40-60 ${ }^{\circ} \mathrm{C}$ )-EtOAc] of the residue afforded 23 , isolated as a white foam $(3.4 \mathrm{~g}, 89 \%) ;[\alpha]_{\mathrm{D}}-86^{\circ}(c) ; R_{f}$ 0.37. NMR data $\left(\mathrm{CDCl}_{3}\right):{ }^{1} \mathrm{H}, \delta$ 7.39-7.29 $(\mathrm{m}, 5 \mathrm{H}, \mathrm{Ph}), 5.872\left(\mathrm{~m}, 1 \mathrm{H}, \mathrm{H}_{2} \mathrm{C}=\mathrm{CHCH}\right), 5.505$ and $5.326(2 \mathrm{~m}$, each $1 \mathrm{H}$, $\left.H_{2} \mathrm{C}=\mathrm{CHCH}\right), 5.166\left(\mathrm{t}, 1 \mathrm{H}, J_{3^{\prime}, 2^{\prime}}=J_{3^{\prime}, 4^{\prime}}=7.7 \mathrm{~Hz}, \mathrm{H}-3^{\prime}\right), 4.913\left(\mathrm{~d}, 1 \mathrm{H}, J_{1^{\prime}, 2^{\prime}}, 5.6 \mathrm{~Hz}\right.$, $\left.\mathrm{H}-1^{\prime}\right), 4.738$ (s, $2 \mathrm{H}, \mathrm{PhCH} \mathrm{H}_{2} \mathrm{O}$ ), 4.302 (dd, $1 \mathrm{H}, J_{5^{\prime} e q, 4^{\prime}} 4.6, J_{5^{\prime} e q, 5^{\prime} a x}-12.0 \mathrm{~Hz}$, H-5'eq), 4.276 (s, $1 \mathrm{H}, \mathrm{H}-1), 4.177$ (dd, $\left.1 \mathrm{H}, J_{6 \mathrm{a}, 5} 4.8, J_{6 \mathrm{a}, 6 \mathrm{~b}}-10.4 \mathrm{~Hz}, \mathrm{H}-6 \mathrm{a}\right), 4.129$ $\left(\mathrm{d}, 1 \mathrm{H}, J_{2,3} 3.0 \mathrm{~Hz}, \mathrm{H}-2\right), 3.861\left(\mathrm{t}, 1 \mathrm{H}, J_{4,3}=J_{4,5}=9.6 \mathrm{~Hz}, \mathrm{H}-4\right), 3.657\left(\mathrm{t}, 1 \mathrm{H}, J_{6 \mathrm{~b}, 5}\right.$ $10.3 \mathrm{~Hz}, \mathrm{H}-6 \mathrm{~b}$ ), 3.506 (dd, $\left.1 \mathrm{H}, J_{3,4} 9.9 \mathrm{~Hz}, \mathrm{H}-3\right), 3.441$ (s, $3 \mathrm{H}, \mathrm{CH}_{3} \mathrm{O}$ ), 3.371 (dd, 1 $\mathrm{H}, J_{5^{\prime} a x, 4^{\prime}} 7.4 \mathrm{~Hz}, \mathrm{H}-5^{\prime} \mathrm{ax}$ ), 3.181 (m, $\left.1 \mathrm{H}, \mathrm{H}-5\right), 2.063,2.054$, and 2.028 (3 s, each 3 $\mathrm{H}, 3 \mathrm{Ac}) ;{ }^{13} \mathrm{C}, \delta 169.8\left(\mathrm{COCH}_{3}\right), 138.0$ and $128.2-127.5\left(C_{6} \mathrm{H}_{5} \mathrm{CH}_{2} \mathrm{O}\right), 133.6$ $\left(\mathrm{H}_{2} \mathrm{C}=\mathrm{CHCH}\right), 118.8 \quad\left(\mathrm{H}_{2} \mathrm{C}=\mathrm{CHCH}\right), 102.3$ and $100.6(2 \mathrm{C})\left(\mathrm{C}-1,1^{\prime}\right.$ and $\left.\mathrm{H}_{2} \mathrm{C}=\mathrm{CHCH}\right), 77.7,76.0,74.8,70.2,69.9,68.9$, and $67.4\left(\mathrm{C}-2,3,4,5,2^{\prime}, 3^{\prime}, 4^{\prime}\right), 71.4$, 68.1, and $61.4\left(\mathrm{C}-6,5^{\prime}\right.$ and $\left.\mathrm{PhCH}_{2} \mathrm{O}\right), 56.8\left(\mathrm{CH}_{3} \mathrm{O}\right), 20.7\left(\mathrm{COCH}_{3}\right)$. Anal. Calcd for $\mathrm{C}_{28} \mathrm{H}_{36} \mathrm{O}_{13}: \mathrm{C}, 57.93 ; \mathrm{H}, 6.25$. Found: $\mathrm{C}, 57.67 ; \mathrm{H}, 6.28$.

Methyl 6-O-allyl-3-O-benzyl-2-O-(2,3,4-tri-O-acetyl- $\beta$-D-xylopyranosyl)- $\beta$-Dmannopyranoside (24).-A solution of $23(3.2 \mathrm{~g}, 5.5 \mathrm{mmol})$ and sodium cyanoborohydride $(4.2 \mathrm{~g}, 67 \mathrm{mmol})$ in tetrahydrofuran $(100 \mathrm{~mL})$ containing $3 \mathrm{~A}$ molecular sieves $\left(7.5 \mathrm{~g}\right.$ ) was stirred for $30 \mathrm{~min}$ under $\mathrm{N}_{2}$. A saturated solution of $\mathrm{HCl}$ in diethyl ether was added until the evolution of gas ceased, and the mixture was stirred for $2 \mathrm{~h}$. TLC (95:5 $\mathrm{CH}_{2} \mathrm{Cl}_{2}$-acetone) then showed the conversion of 23 ( $R_{f}$ $0.45)$ into $24\left(R_{f} 0.23\right)$. The mixture was diluted with $\mathrm{CH}_{2} \mathrm{Cl}_{2}(250 \mathrm{~mL})$, filtered through Celite, washed with water, aq $5 \% \mathrm{NaHCO}_{3}$, and water, dried $\left(\mathrm{MgSO}_{4}\right)$, filtered, and concentrated, and $\mathrm{MeOH}(3 \times 25 \mathrm{~mL})$ was evaporated from the residue. Column chromatography $\left(9: 1 \mathrm{CH}_{2} \mathrm{Cl}_{2}\right.$-acetone) of the residue gave 24, isolated as a white solid $(2.2 \mathrm{~g}, 69 \%) ;[\alpha]_{\mathrm{D}}-111^{\circ}(c 1) ; R_{f} 0.42$. NMR data $\left(\mathrm{CDCl}_{3}\right):{ }^{1} \mathrm{H}, \delta 7.37-7.26(\mathrm{~m}, 5 \mathrm{H}, \mathrm{Ph}), 5.897\left(\mathrm{~m}, 1 \mathrm{H}, \mathrm{H}_{2} \mathrm{C}=\mathrm{CHCH}_{2} \mathrm{O}\right), 5.280$ and 
$5.174\left(2 \mathrm{~m}\right.$, each $\left.1 \mathrm{H}, \mathrm{H}_{2} \mathrm{C}=\mathrm{CHCH}_{2} \mathrm{O}\right), 5.181\left(\mathrm{t}, 1 \mathrm{H}, J_{3^{\prime}, 4^{\prime}}, 8.0 \mathrm{~Hz}, \mathrm{H}-3^{\prime}\right), 5.002(\mathrm{dd}$, $\left.1 \mathrm{H}, J_{2^{\prime}, 3^{\prime}}, 8.2 \mathrm{~Hz}, \mathrm{H}-2^{\prime}\right), 4.924\left(\mathrm{~m}, 1 \mathrm{H}, J_{4^{\prime}, 5^{\prime} e q} 4.8, J_{4^{\prime}, 5^{\prime} \mathrm{ax}} 8.0 \mathrm{~Hz}, \mathrm{H}-4^{\prime}\right), 4.899$ (d, 1 $\left.\mathrm{H}, J_{1^{\prime}, 2^{\prime}} 6.3 \mathrm{~Hz}, \mathrm{H}-1^{\prime}\right), 4.803$ and $4.494\left(2 \mathrm{~d}\right.$, each $\left.1 \mathrm{H}, \mathrm{PhCH} \mathrm{H}_{2} \mathrm{O}\right), 4.260(\mathrm{~s}, 1 \mathrm{H}$, $\mathrm{H}-1$ ), 4.226 (dd, $\left.1 \mathrm{H}, J_{5^{\prime} e q, 5^{\prime} a x}-12.9 \mathrm{~Hz}, \mathrm{H}-5^{\prime} e q\right), 4.148$ (d, $1 \mathrm{H}, J_{2,3} 2.9 \mathrm{~Hz}, \mathrm{H}-2$ ), 4.049 and $4.031\left(2 \mathrm{~m}\right.$, each $\left.1 \mathrm{H}, \mathrm{H}_{2} \mathrm{C}=\mathrm{CHCH}_{2} \mathrm{O}\right), 3.776\left(\mathrm{dd}, 1 \mathrm{H}, J_{6 \mathrm{a}, 5} 3.4, J_{6 \mathrm{a}, 6 \mathrm{~b}}\right.$ $-10.5 \mathrm{~Hz}, \mathrm{H}-6 \mathrm{a}$ ), 3.735 (m, $1 \mathrm{H}, \mathrm{H}-4$ ), 3.620 (dd, $1 \mathrm{H}, J_{6 \mathrm{~b}, 5} 6.4 \mathrm{~Hz}, \mathrm{H}-6 \mathrm{~b}$ ), 3.465 (s, $3 \mathrm{H}, \mathrm{CH}_{3} \mathrm{O}$ ), 3.287 (dd, $1 \mathrm{H}, J_{3,4} 9.3 \mathrm{~Hz}, \mathrm{H}-3$ ), 2.538 (d, $1 \mathrm{H}, J_{\mathrm{HO}-4,4} 1.9 \mathrm{~Hz}, \mathrm{HO}-4$ ), $2.049(\mathrm{~s}, 9 \mathrm{H}, 3 \mathrm{Ac}) ;{ }^{13} \mathrm{C}, \delta 169.8(2 \mathrm{C})$ and $169.4\left(3 \mathrm{COCH}_{3}\right), 137.5$ and 128.4-127.8 $\left(\mathrm{C}_{6} \mathrm{H}_{5} \mathrm{CH}_{2} \mathrm{O}\right), 134.5\left(\mathrm{H}_{2} \mathrm{C}=\mathrm{CHCH}_{2} \mathrm{O}\right), 116.9\left(\mathrm{H}_{2} \mathrm{C}=\mathrm{CHCH}_{2} \mathrm{O}\right), 101.5$ and 100.6 (C-1,1'), 72.3, 70.6, 70.4, and 61.5 (C-6,5, $\mathrm{PhCH}_{2} \mathrm{O}$, and $\mathrm{H}_{2} \mathrm{C}=\mathrm{CHCH}_{2} \mathrm{O}$ ), $56.6\left(\mathrm{CH}_{3} \mathrm{O}\right), 20.7\left(\mathrm{COCH}_{3}\right)$. Anal. Calcd for $\mathrm{C}_{28} \mathrm{H}_{38} \mathrm{O}_{13}: \mathrm{C}, 57.72 ; \mathrm{H}, 6.57$. Found: C, 57.15; H, 6.42 .

Methyl 4-O-acetyl-6-O-allyl-3-O-benzyl-2-O-(2,3,4-tri-O-acetyl- $\beta$-D-xylopyranosyl)- $\beta$-D-mannopyranoside (25). - A solution of $24(2.1 \mathrm{~g}, 3.6 \mathrm{mmol})$ in 1:1 pyridine-acetic anhydride $(50 \mathrm{~mL})$ was stirred for $16 \mathrm{~h}$, and toluene $(3 \times 20 \mathrm{~mL})$, EtOH $(3 \times 20 \mathrm{~mL})$, and $\mathrm{CH}_{2} \mathrm{Cl}_{2}(3 \times 20 \mathrm{~mL})$ were evaporated from the residue to give 25 , isolated as a yellow solid $(2.2 \mathrm{~g}, 98 \%) ; R_{f} 0.56\left(95: 5 \mathrm{CH}_{2} \mathrm{Cl}_{2}\right.$-acetone). NMR data $\left(\mathrm{CDCl}_{3}\right):{ }^{1} \mathrm{H}, \delta$ 7.35-7.27 (m, $\left.5 \mathrm{H}, \mathrm{Ph}\right), 5.868\left(\mathrm{~m}, 1 \mathrm{H}, \mathrm{H}_{2} \mathrm{C}=\mathrm{CHCH}_{2} \mathrm{O}\right)$, 5.255 and $5.163\left(2 \mathrm{~m}\right.$, each $\left.1 \mathrm{H}, H_{2} \mathrm{C}=\mathrm{CHCH}_{2} \mathrm{O}\right), 5.128\left(\mathrm{t}, 1 \mathrm{H}, J_{4,3}=J_{4,5}=9.6 \mathrm{~Hz}\right.$, $\mathrm{H}-4), 4.716$ and $4.499\left(2 \mathrm{~d}\right.$, each $\left.1 \mathrm{H}, \mathrm{PhCH}_{2} \mathrm{O}\right), 4.442$ (dd, $1 \mathrm{H}, J_{5^{\prime} a x / e q, 4^{\prime}} 3.4$, $J_{5^{\prime} a x, 5^{\prime} e q}-12.0 \mathrm{~Hz}, \mathrm{H}-5^{\prime} a x$ or H-5'eq), $4.281(\mathrm{~s}, 1 \mathrm{H}, \mathrm{H}-1), 4.149\left(\mathrm{~d}, 1 \mathrm{H}, J_{2,3} 3.0\right.$ $\mathrm{Hz}, \mathrm{H}-2), 4.000$ and $3.982\left(2 \mathrm{~m}\right.$, each $\left.1 \mathrm{H}, \mathrm{H}_{2} \mathrm{C}=\mathrm{CHCH} \mathrm{H}_{2} \mathrm{O}\right), 3.469\left(\mathrm{~s}, 3 \mathrm{H}, \mathrm{CH}_{3} \mathrm{O}\right.$ ), 2.141, 2.075, 2.072, and $2.028(4 \mathrm{~s}$, each $3 \mathrm{H}, 4 \mathrm{Ac}) ;{ }^{13} \mathrm{C}, \delta 169.7(3 \mathrm{C})$ and $169.0(4$ $\left.\mathrm{COCH}_{3}\right), 137.6,128.3,127.7$, and $127.5\left(C_{6} \mathrm{H}_{5} \mathrm{CH}_{2} \mathrm{O}\right), 134.4\left(\mathrm{H}_{2} \mathrm{C}=\mathrm{CHCH}_{2} \mathrm{O}\right)$, $117.1\left(\mathrm{H}_{2} \mathrm{C}=\mathrm{CHCH}_{2} \mathrm{O}\right), 101.4$ and $99.0\left(\mathrm{C}-1,1^{\prime}\right), 77.3,73.9,72.8,68.7,68.2$, and 67.8 (2 C) $\left(\mathrm{C}-2,3,4,5,2^{\prime}, 3^{\prime}, 4^{\prime}\right), 72.4,70.7,70.0$, and 59.6 (C-6,5', $\mathrm{PhCH}_{2} \mathrm{O}$, and $\left.\mathrm{H}_{2} \mathrm{C}=\mathrm{CHCH} \mathrm{H}_{2} \mathrm{O}\right), 56.8\left(\mathrm{CH}_{3} \mathrm{O}\right), 20.7\left(\mathrm{COCH}_{3}\right)$. Anal. Calcd for $\mathrm{C}_{30} \mathrm{H}_{40} \mathrm{O}_{14}: \mathrm{C}$, 57.68; $\mathrm{H}, 6.45$. Found: $\mathrm{C}, 57.28 ; \mathrm{H}, 6.53$.

Methyl 4-O-acetyl-3-O-benzyl-2-O-(2,3,4-tri-O-acetyl- $\beta$-D-xylopyranosyl)- $\beta$-Dmannopyranoside (26-a) and methyl 6-O-acetyl-3-O-benzyl-2-O-(2,3,4-tri-O-acetyl$\beta$-D-xylopyranosyl) $-\beta$-D-mannopyranoside (26-b). - To a mixture of 25 (746 mg, 1.2 $\mathrm{mmol})$ and 1,4-diazabicyclo[2.2.2]octane $(100 \mathrm{mg})$ in $8: 3: 1 \mathrm{EtOH}$-toluene-water $(40 \mathrm{~mL})$ was added tris(triphenylphosphine)rhodium(I) chloride $(170 \mathrm{mg})$, and the mixture was boiled under reflux for $2 \mathrm{~h}$, then cooled, and concentrated. A solution of the residue in acetone $(45 \mathrm{~mL})$ and $\mathrm{M} \mathrm{HCl}(5 \mathrm{~mL})$ was boiled under reflux for $30 \mathrm{~min}$, when TLC (9:1 $\mathrm{CH}_{2} \mathrm{Cl}_{2}$-acetone) showed the conversion of the propenyl analogue of $25\left(R_{f} 0.88\right)$ into 26-a $\left(R_{f} 0.14\right)$ to be complete. The mixture was neutralised with aq $5 \% \mathrm{NaHCO}_{3}$, concentrated, diluted with $\mathrm{CH}_{2} \mathrm{Cl}_{2}(100 \mathrm{~mL})$, washed with water, dried $\left(\mathrm{MgSO}_{4}\right)$, filtered, and concentrated. Column chromatography $\left(8: 2 \mathrm{CH}_{2} \mathrm{Cl}_{2}\right.$-acetone) of the residue first gave $26-\mathrm{b}(280 \mathrm{mg}, 40 \%) ; R_{f}$ 0.73 , then 26-a $(371 \mathrm{mg}, 53 \%) ;[\alpha]_{\mathrm{D}}-108^{\circ}(c 1) ; R_{f} 0.57$, both isolated as a white solid. NMR data $\left(\mathrm{CDCl}_{3}\right)$ for $26-\mathrm{a}:{ }^{1} \mathrm{H}, \delta$ 7.71-7.31 (m, $\left.5 \mathrm{H}, \mathrm{Ph}\right), 5.243\left(\mathrm{t}, 1 \mathrm{H}, J_{4,5}\right.$ $9.8 \mathrm{~Hz}, \mathrm{H}-4), 4.730$ and 4.505 ( $2 \mathrm{~d}$, each $1 \mathrm{H}, \mathrm{PhCH}_{2} \mathrm{O}$ ), 4.433 and 3.402 ( $2 \mathrm{dd}$, 
each $1 \mathrm{H}, J_{5^{\prime} a x / e q, 4^{\prime}} 3.5$ and $4.7, \mathrm{~J}_{5^{\prime} a x, 5^{\prime} e q}-12.6$ and $\left.-12.4 \mathrm{~Hz}, \mathrm{H}-5^{\prime} e q, 5^{\prime} a x\right), 4.317$ (s, $1 \mathrm{H}, \mathrm{H}-1$ ), 4.164 (d, $1 \mathrm{H}, J_{2,3} 2.9 \mathrm{~Hz}, \mathrm{H}-2$ ), 3.487 (dd, $1 \mathrm{H}, J_{3,4} 9.8 \mathrm{~Hz}, \mathrm{H}-3$ ), 3.475 (s, $3 \mathrm{H}, \mathrm{CH}_{3} \mathrm{O}$ ), 3.312 (m, $\left.1 \mathrm{H}, \mathrm{H}-5\right), 2.481$ (m, $\left.1 \mathrm{H}, \mathrm{HO}-6\right), 2.135,2.082$, 2.069, and $2.051(4 \mathrm{~s}$, each $3 \mathrm{H}, 4 \mathrm{Ac}) ;{ }^{13} \mathrm{C}, \delta 169.9$ and $169.2\left(\mathrm{COCH}_{3}\right), 137.5$, $128.3,127.7$, and $127.4\left(C_{6} \mathrm{H}_{5} \mathrm{CH}_{2} \mathrm{O}\right), 101.5$ and $99.2\left(\mathrm{C}-1,1^{\prime}\right), 77.3,74.5,72.8,68.5$, $68.0\left(2 \mathrm{C}\right.$ ), and $67.5\left(\mathrm{C}-2,3,4,5,2^{\prime}, 3^{\prime}, 4^{\prime}\right), 70.6,61.5$, and $59.7\left(\mathrm{C}-6,5^{\prime}\right.$ and $\mathrm{PhCH}_{2} \mathrm{O}$ ), $56.9\left(\mathrm{CH}_{3} \mathrm{O}\right), 20.8(3 \mathrm{C})$ and $20.6\left(4 \mathrm{COCH}_{3}\right)$. NMR data $\left(\mathrm{CDCl}_{3}\right)$ for $26-\mathrm{b}:{ }^{1} \mathrm{H}, \delta$ 7.39-7.27 (m, $5 \mathrm{H}, \mathrm{Ph}$ ), 5.212 (t, $1 \mathrm{H}, J_{3^{\prime}, 4^{\prime}} 8.8 \mathrm{~Hz}, \mathrm{H}-3^{\prime}$ ), 5.026 (dd, $1 \mathrm{H}, J_{2^{\prime}, 3^{\prime}} 9.0$ $\left.\mathrm{Hz}, \mathrm{H}-2^{\prime}\right), 4.957\left(\mathrm{~m}, 1 \mathrm{H}, J_{4^{\prime}, 5^{\prime} a x} 8.8, J_{4^{\prime}, 5^{\prime} e q} 5.1 \mathrm{~Hz}, \mathrm{H}^{\prime} 4^{\prime}\right), 4.863\left(\mathrm{~d}, 1 \mathrm{H}, J_{1^{\prime}, 2^{\prime}} 7.0\right.$ $\left.\mathrm{Hz}, \mathrm{H}-1^{\prime}\right), 4.809$ and $4.479\left(2 \mathrm{~d}\right.$, each $\left.1 \mathrm{H}, \mathrm{PhCH}_{2} \mathrm{O}\right), 4.256(\mathrm{~s}, 1 \mathrm{H}, \mathrm{H}-1), 4.174$ (dd, $1 \mathrm{H}, \mathrm{H}-5^{\prime} e q$ ), 4.158 (d, $1 \mathrm{H}, J_{2,3} 3.0 \mathrm{~Hz}, \mathrm{H}-2$ ), 3.759 (dt, $1 \mathrm{H}, J_{4,5} 9.5 \mathrm{~Hz}, \mathrm{H}-4$ ), 3.459 (s, $3 \mathrm{H}, \mathrm{CH}_{3} \mathrm{O}$ ), 3.336 (dd, $1 \mathrm{H}, J_{5^{\prime} a x, 5^{\prime} e q}-11.8 \mathrm{~Hz}, \mathrm{H}-5^{\prime} \mathrm{ax}$ ), 3.283 (dd, $1 \mathrm{H}$, $\left.J_{3,4} 9.2 \mathrm{~Hz}, \mathrm{H}-3\right), 2.512\left(\mathrm{~d}, 1 \mathrm{H}, J_{\mathrm{HO}-4,4} 2.6 \mathrm{~Hz}, \mathrm{HO}-4\right), 2.124$ and $2.038(2 \mathrm{~s}, 3$ and 9 $\mathrm{H}, 4$ Ac). Anal. Calcd for $\mathrm{C}_{27} \mathrm{H}_{36} \mathrm{O}_{14}: \mathrm{C}, 55.48 ; \mathrm{H}, 6.21$. Found 26-a: $\mathrm{C}, 55.36 ; \mathrm{H}$, 6.26 .

Methyl 4-O-acetyl-3-O-benzyl-6-O-(2,3,4,6-tetra-O-acetyl- $\alpha$-D-mannopyranosyl)-2O-(2,3,4-tri-O-acetyl- $\beta$-D-xylopyranosyl)- $\beta$-D-mannopyranoside (28).-A solution of 26-a (309 mg, $0.53 \mathrm{mmol}$ ) and 2,3,4,6-tetra- $O$-acetyl- $\alpha$-D-mannopyranosyl trichloroacetimidate ${ }^{8}(27 ; 345 \mathrm{mg}, 0.70 \mathrm{mmol})$ in $\mathrm{CH}_{2} \mathrm{Cl}_{2}(10 \mathrm{~mL})$, containing powdered $4 \mathrm{~A}$ molecular sieves $(1 \mathrm{~g})$, was stirred for $30 \mathrm{~min}$ under $\mathrm{N}_{2}$. A solution of trimethylsilyl triflate $(50 \mu \mathrm{L})$ in $\mathrm{CH}_{2} \mathrm{Cl}_{2}(5 \mathrm{~mL})$ was added dropwise at $-40^{\circ} \mathrm{C}$, and after $1 \mathrm{~h}$, when TLC showed the disappearance of 26-a and a new product $28\left(R_{f} 0.53,9: 1\right.$ $\mathrm{CH}_{2} \mathrm{Cl}_{2}$-acetone), pyridine $(1 \mathrm{~mL})$ was added, the mixture was filtered through Celite and concentrated, and toluene $(3 \times 10 \mathrm{~mL})$ was evaporated from the residue. Column chromatography $\left(9: 1 \mathrm{CH}_{2} \mathrm{Cl}_{2}-\right.$ acetone) of the residue gave 28 , isolated as a white solid $(359 \mathrm{mg}, 77 \%)$; $[\alpha]_{\mathrm{D}} \quad 65^{\circ}\left(c\right.$ 1.5). NMR data $\left(\mathrm{CDCl}_{3}\right)$ : ${ }^{1} \mathrm{H}, \delta$ 7.36-7.25 (m, $5 \mathrm{H}, \mathrm{Ph}$ ), 5.199 (dd, $\left.1 \mathrm{H}, J_{2^{\prime \prime}, 3^{\prime \prime}} 3.0 \mathrm{~Hz}, \mathrm{H}-2^{\prime \prime}\right), 4.790$ (d, $1 \mathrm{H}$, $\left.J_{1^{\prime \prime}, 2^{\prime \prime}} 1.8 \mathrm{~Hz}, \mathrm{H}-1^{\prime \prime}\right), 4.718$ and $4.506\left(2 \mathrm{~d}\right.$, each $\left.1 \mathrm{H}, \mathrm{PhCH} \mathrm{H}_{2} \mathrm{O}\right), 4.401$ and 3.398 (2 dd, each $1 \mathrm{H}, J_{5^{\prime} e q / a x, 4^{\prime}} 3.5$ and $\left.4.9, J_{5^{\prime} e q, 5^{\prime} a x}-12.3 \mathrm{~Hz}, \mathrm{H}-5^{\prime} a x, 5^{\prime} e q\right), 4.311(\mathrm{~s}, 1 \mathrm{H}$, $\mathrm{H}-1$ ), 4.157 (d, $\left.1 \mathrm{H}, J_{2,3} 3.0 \mathrm{~Hz}, \mathrm{H}-2\right), 3.481$ (s, $3 \mathrm{H}, \mathrm{CH}_{3} \mathrm{O}$ ), 2.147, 2.113, 2.104, 2.066, 2.052, 2.014, and $1.978(7 \mathrm{~s}, 3,3,3,6,3,3,3 \mathrm{H}, 8 \mathrm{Ac}) ;{ }^{13} \mathrm{C}, \delta 170.6-168.8$ $\left(\mathrm{COCH}_{3}\right), 137.5$ and $128.3-127.5\left(C_{6} \mathrm{H}_{5} \mathrm{CH}_{2} \mathrm{O}\right), 101.3,99.2$, and $96.9\left(\mathrm{C}-1,1^{\prime}, 1^{\prime \prime}\right)$, $77.5,72.9,72.7,69.3,68.9,68.7,68.5(2 \mathrm{C}), 68.0(2 \mathrm{C})$, and 65.9 (C2,3,4,5, $\left.2^{\prime}, 3^{\prime}, 4^{\prime}, 2^{\prime \prime}, 3^{\prime \prime}, 4^{\prime \prime}, 5^{\prime \prime}\right), 70.9,67.6,62.1$, and $59.8\left(\mathrm{C}-6,5^{\prime}, 6^{\prime \prime}\right.$ and $\left.\mathrm{PhCH}_{2} \mathrm{O}\right), 56.7$ $\left(\mathrm{CH}_{3} \mathrm{O}\right), 20.7\left(\mathrm{COCH}_{3}\right)$. Anal. Calcd for $\mathrm{C}_{41} \mathrm{H}_{54} \mathrm{O}_{23}: \mathrm{C}, 53.83 ; \mathrm{H}, 5.95$. Found: $\mathrm{C}$, 53.29; $\mathrm{H}, 6.01$.

Methyl 4-O-acetyl-6-O-(2,3,4,6-tetra-O-acetyl- $\alpha$-D-mannopyranosyl)-2-O-(2,3,4tri-O-acetyl- $\beta$-D-xylopyranosyl)- $\beta$-D-mannopyranoside (29).-To a solution of 28 $(330 \mathrm{mg}, 0.37 \mathrm{mmol})$ in $1: 1 \mathrm{EtOH}-\mathrm{EtOAc}(12 \mathrm{~mL})$ were added acetic acid $(0.3$ $\mathrm{mL}$ ) and $10 \% \mathrm{Pd}-\mathrm{C}(250 \mathrm{mg})$. Hydrogenolysis, and work-up as described for 12 , gave 29, isolated as a white solid (300 $\mathrm{mg}, 98 \%) ; R_{f} 0.11\left(9: 1 \mathrm{CH}_{2} \mathrm{Cl}_{2}\right.$-acetone). NMR data $\left(\mathrm{CDCl}_{3}\right):{ }^{1} \mathrm{H}, \delta 5.228\left(\mathrm{dd}, 1 \mathrm{H}, J_{2^{\prime \prime}, 3^{\prime \prime}} 3.2 \mathrm{~Hz}, \mathrm{H}-2^{\prime \prime}\right), 4.944\left(\mathrm{dd}, 1 \mathrm{H}, J_{2^{\prime}, 3^{\prime}}\right.$ $8.6 \mathrm{~Hz}, \mathrm{H}-2^{\prime}$ ), $4.836\left(\mathrm{~d}, 1 \mathrm{H}, J_{1^{\prime \prime}, 2^{\prime \prime}} 1.8 \mathrm{~Hz}, \mathrm{H}-1^{\prime \prime}\right), 4.750\left(\mathrm{~d}, 1 \mathrm{H}, J_{1^{\prime}, 2^{\prime}} 6.7 \mathrm{~Hz}, \mathrm{H}-1^{\prime}\right.$ ), 
4.385 (s, $1 \mathrm{H}, \mathrm{H}-1), 4.001$ (d, $\left.1 \mathrm{H}, J_{2,3} 3.2 \mathrm{~Hz}, \mathrm{H}-2\right), 3.504\left(\mathrm{~s}, 3 \mathrm{H}, \mathrm{CH}_{3} \mathrm{O}\right.$ ), 3.388 (dd, $1 \mathrm{H}, J_{5^{\prime} a x, 4^{\prime}} 8.8, J_{5^{\prime} a x, 5^{\prime} e q}-11.9 \mathrm{~Hz}, \mathrm{H}-5^{\prime} a x$ ), 2.156, 2.121, 2.113, 2.064, 2.041, 2.010 , and $1.986(7 \mathrm{~s}, 3,3,3,3,6,3,3 \mathrm{H}, 8 \mathrm{Ac}) ;{ }^{13} \mathrm{C}, \delta 170.5-169.4\left(\mathrm{COCH}_{3}\right), 100.9(2$ C) and $96.8\left(\mathrm{C}-1,1^{\prime}, 1^{\prime \prime}\right), 77.9,72.6,71.0,70.3,70.0(2 \mathrm{C}), 69.3,68.8,68.3(2 \mathrm{C})$, and $65.8\left(\mathrm{C}-2,3,4,5,2^{\prime}, 3^{\prime}, 4^{\prime}, 2^{\prime \prime}, 3^{\prime \prime}, 4^{\prime \prime}, 5^{\prime \prime}\right), 66.8,62.0$, and $61.7\left(\mathrm{C}-6,5^{\prime}, 6^{\prime \prime}\right), 56.8\left(\mathrm{CH}_{3} \mathrm{O}\right)$, $20.5\left(\mathrm{COCH}_{3}\right)$. Anal. Calcd for $\mathrm{C}_{34} \mathrm{H}_{48} \mathrm{O}_{23}: \mathrm{C}, 49.52 ; \mathrm{H}$, 5.87. Found: C, 49.16; $\mathrm{H}$, 5.81 .

Methyl 4-O-acetyl-6-O-(2,3,4,6-tetra-O-acetyl- $\alpha$-D-mannopyranosyl)-3-O-(2,4,6tri-O-acetyl-3-O-methyl- $\alpha$-D-mannopyranosyl)-2-O-(2,3,4-tri-O-acetyl- $\beta$-D-xylopyranosyl)- $\beta$-D-mannopyranoside (30).-A solution of $29(109 \mathrm{mg}, 0.13 \mathrm{mmol})$ and 18 (140 mg, $0.3 \mathrm{mmol})$ in $\mathrm{CH}_{2} \mathrm{Cl}_{2}(10 \mathrm{~mL})$ containing powdered $4 \mathrm{~A}$ molecular sieves $(1 \mathrm{~g})$ was stirred for $30 \mathrm{~min}$ under $\mathrm{N}_{2}$. A solution of trimethylsilyl triflate $(30 \mu \mathrm{L})$ in $\mathrm{CH}_{2} \mathrm{Cl}_{2}(5 \mathrm{~mL})$ was added dropwise at $-40^{\circ} \mathrm{C}$, and after $1.5 \mathrm{~h}$, when TLC showed the disappearance of 29 and a new compound $30\left(R_{f} 0.49,9: 1 \mathrm{CH}_{2} \mathrm{Cl}_{2}\right.$-acetone), pyridine $(1 \mathrm{~mL})$ was added. The mixture was filtered through Celite and concentrated, and toluene $(3 \times 10 \mathrm{~mL})$ was evaporated from the residue. Column chromatography $\left(9: 1 \mathrm{CH}_{2} \mathrm{Cl}_{2}\right.$-acetone) of the residue afforded 30 , isolated as a white solid (90 mg, 61\%); $[\alpha]_{\mathrm{D}}-19^{\circ}(c)$ ). NMR data $\left(\mathrm{CDCl}_{3}\right):{ }^{1} \mathrm{H}, \delta 4.926\left(\mathrm{~d}, 1 \mathrm{H}, J_{1^{\prime \prime}, 2^{\prime \prime}}\right.$ or $J_{1^{\prime \prime \prime}, 2^{\prime \prime \prime}} 1.7 \mathrm{~Hz}, \mathrm{H}-1^{\prime \prime}$ or $\left.\mathrm{H}-1^{\prime \prime \prime}\right), 4.367(\mathrm{~s}, 1 \mathrm{H}, \mathrm{H}-1), 4.308\left(\mathrm{dd}, 1 \mathrm{H}, J_{5^{\prime} e q, 4^{\prime}} 5.2 \mathrm{~Hz}\right.$, $\left.\mathrm{H}-5^{\prime} e q\right), 4.002\left(\mathrm{~d}, 1 \mathrm{H}, J_{2,3} 3.0 \mathrm{~Hz}, \mathrm{H}-2\right), 3.505$ and $3.386\left(2 \mathrm{~s}\right.$, each $3 \mathrm{H}, 2 \mathrm{CH}_{3} \mathrm{O}$ ), 3.346 (dd, $1 \mathrm{H}, J_{5^{\prime} u x, 4^{\prime}} 8.2, J_{5^{\prime} u x, 5^{\prime} e q}-11.6 \mathrm{~Hz}, \mathrm{H}-5^{\prime} a x$ ), 2.155, 2.128, 2.123, 2.085 , 2.080, 2.078, 2.047, 2.046, 2.016, and $1.984(10 \mathrm{~s}, 6,3,3,3,3,3,3,3,3,3 \mathrm{H}, 11 \mathrm{Ac}) ;{ }^{13} \mathrm{C}, \delta$ 170.6-168.9 $\left(\mathrm{COCH}_{3}\right), 101.1,101.0,99.9$, and $96.9\left(\mathrm{C}-1,1^{\prime}, 1^{\prime \prime}, 1^{\prime \prime \prime}\right), 67.3,63.0,62.1$, and $61.3\left(\mathrm{C}-6,5^{\prime}, 6^{\prime \prime}, 6^{\prime \prime \prime}\right), 58.0$ and $56.8\left(2 \mathrm{CH}_{3} \mathrm{O}\right), 20.5\left(\mathrm{COCH}_{3}\right)$. Anal. Calcd for $\mathrm{C}_{47} \mathrm{H}_{66} \mathrm{O}_{31}: \mathrm{C}, 50.09 ; \mathrm{H}, 5.90$. Found: $\mathrm{C}, 49.15 ; \mathrm{H}, 5.82$.

Methyl 6-O- $\alpha$-D-mannopyranosyl-3-O-(3-O-methyl- $\alpha$-D-mannopyranosyl)-2-O- $\beta$ D-xylopyranosyl- $\beta$-D-mannopyranoside (3). -Deacetylation of $\mathbf{3 0}(24 \mathrm{mg}, 22 \mu \mathrm{mol})$, as described for 2 , gave 3 , isolated as a white powder $(14 \mathrm{mg}, 96 \%) ;[\alpha]_{\mathrm{D}}+2^{\circ}(c$ $\left.0.5, \mathrm{H}_{2} \mathrm{O}\right) ; R_{f} 0.47$ (2:1:1 1-butanol-MeOH-water). ${ }^{13} \mathrm{C}$ NMR data $\left(\mathrm{D}_{2} \mathrm{O}\right): \delta$ 106.5, 103.3, 102.6, and $100.9\left(\mathrm{C}-1,1^{\prime}, 1^{\prime \prime}, 1^{\prime \prime \prime}\right), 58.2$ and $57.4\left(2 \mathrm{CH}_{3} \mathrm{O}\right)$. For ${ }^{1} \mathrm{H}$ NMR data, see Table I.

Methyl 4-O-acetyl-3-O-benzyl-6-O-(2,4,6-tri-O-acetyl-3-O-methyl- $\alpha$-D-mannopyranosyl)-2-O-(2,3,4-tri-O-acetyl- $\beta$-D-xylopyranosyl)- $\beta$-D-mannopyranoside (31). -Compound 25 ( $200 \mathrm{mg}, 0.32 \mathrm{mmol}$ ) was converted into $26 \mathrm{a}$ as described above, but the residue after work-up was not purified by column chromatography. A solution of the residue (26-a) and $18(250 \mathrm{mg}, 0.54 \mathrm{mmol})$ in $\mathrm{CH}_{2} \mathrm{Cl}_{2}(10 \mathrm{~mL})$ containing powdered $4 \mathrm{~A}$ molecular sieves $(1 \mathrm{~g})$ was stirred for 30 min under $\mathrm{N}_{2}$. A solution of trimethylsilyl triflate $(50 \mu \mathrm{L})$ in $\mathrm{CH}_{2} \mathrm{Cl}_{2}(5 \mathrm{~mL})$ was added dropwise at $-40^{\circ} \mathrm{C}$, and after $1 \mathrm{~h}$, when TLC showed the disappearance of 26-a and a new product $31\left(R_{f} 0.23,9: 1 \mathrm{CH}_{2} \mathrm{Cl}_{2}\right.$-acetone), pyridine $(1 \mathrm{~mL})$ was added, the mixture was filtered through Celite and concentrated, and tolucnc $(3 \times 10 \mathrm{~mL})$ was evaporated from the residue. Column chromatography $\left(9: 1 \mathrm{CH}_{2} \mathrm{Cl}_{2}\right.$-acetone) of the residue gave 31, isolated as a white solid (155 mg, 51\%); $[\alpha]_{D}-63^{\circ}(c 0.3)$. 
NMR data $\left(\mathrm{CDCl}_{3}\right):{ }^{1} \mathrm{H}, \delta 7.37-7.27(\mathrm{~m}, 5 \mathrm{H}, \mathrm{Ph}), 5.371\left(\mathrm{dd}, 1 \mathrm{H}, J_{2^{\prime \prime}, 3^{\prime \prime}} 3.4 \mathrm{~Hz}\right.$, H-2"), 4.995 (dd, $1 \mathrm{H}, J_{2^{\prime}, 3^{\prime}} 6.5 \mathrm{~Hz}, \mathrm{H}-2^{\prime}$ ), 4.947 (d, $1 \mathrm{H}, J_{1^{\prime}, 2^{\prime}} 4.7 \mathrm{~Hz}, \mathrm{H}-1^{\prime}$ ), 4.811 (d, $\left.1 \mathrm{H}, J_{1^{\prime \prime} 2^{\prime \prime}} 1.8 \mathrm{~Hz}, \mathrm{H}-1^{\prime \prime}\right), 4.727$ and 4.480 ( $2 \mathrm{~d}$, each $1 \mathrm{H}, \mathrm{PhCH} \mathrm{H}_{2} \mathrm{O}$ ), 4.298 (s, 1 $\mathrm{H}, \mathrm{H}-1), 4.260$ (dd, $\left.1 \mathrm{H}, J_{5^{\prime} e q, 4^{\prime}} 5.5, J_{5^{\prime} e q, 5^{\prime} a x}-12.3 \mathrm{~Hz}, \mathrm{H}-5^{\prime} e q\right), 4.167$ (d, $1 \mathrm{H}, J_{2,3}$ $3.0 \mathrm{~Hz}, \mathrm{H}-2), 3.481$ and $3.365\left(2 \mathrm{~s}\right.$, each $3 \mathrm{H}, 2 \mathrm{CH}_{3} \mathrm{O}$ ), 2.134, 2.099, 2.083, 2.078, 2.059, and $2.034(6 \mathrm{~s}, 3,3,3,6,3,3 \mathrm{H}, 7 \mathrm{Ac}) ;{ }^{13} \mathrm{C}, \delta 170.3-168.8\left(\mathrm{COCH}_{3}\right), 137.5$, 128.3, and 127.8-127.6 $\left(C_{6} \mathrm{H}_{5} \mathrm{CH}_{2} \mathrm{O}\right), 101.6,99.8$, and $97.4\left(\mathrm{C}-1,1^{\prime}, 1^{\prime \prime}\right), 67.4,70.6$, 66.7, 62.5, and $60.4\left(\mathrm{C}-6,5^{\prime}, 6^{\prime \prime}\right.$ and $\left.\mathrm{PhCH}_{2} \mathrm{O}\right), 57.7$ and $56.7\left(2 \mathrm{CH}_{3} \mathrm{O}\right), 20.7$ $\left(\mathrm{COCH}_{3}\right)$.

Methyl 4-O-acetyl-6-O-(2,4,6-tri-O-acetyl-3-O-methyl- $\alpha$-D-mannopyranosyl)-2-O(2,3,4-tri-O-acetyl- $\beta$-D-xylopyransyl)- $\beta$-D-mannopyranoside (32). - To a solution of 31 (145 $\mathrm{mg}, 0.16 \mathrm{mmol})$ in $1: 1 \mathrm{EtOH}-\mathrm{EtOAc}(16 \mathrm{~mL})$ were added acetic acid $(0.4$ $\mathrm{mL})$ and $10 \% \mathrm{Pd}-\mathrm{C}(125 \mathrm{mg})$. Hydrogenolysis, and work-up as described for 12, gave 32, isolated as a white solid (127 $\mathrm{mg}, 98 \%) ; R_{f} 0.17\left(9: 1 \mathrm{CH}_{2} \mathrm{Cl}_{2}\right.$-acetone). ${ }^{13} \mathrm{C}$ NMR data $\left(\mathrm{CDCl}_{3}\right): \delta 170.6,170.3,170.0,169.9,169.8,169.6$, and $169.0(7$ $\left.\mathrm{COCH}_{3}\right), 101.4,101.2$, and $97.5\left(\mathrm{C}-1,1^{\prime}, 1^{\prime \prime}\right), 77.7,76.4,72.5,71.3,70.8,70.1,69.3$, 68.6 (2 C), 67.9, and $67.4\left(\mathrm{C}-2,3,4,5,2^{\prime}, 3^{\prime}, 4^{\prime}, 2^{\prime \prime}, 3^{\prime \prime}, 4^{\prime \prime}, 5^{\prime \prime}\right), 66.1,62.5$, and 62.1 $\left(\mathrm{C}-6,5^{\prime}, 6^{\prime \prime}\right), 57.7$ and $56.8\left(2 \mathrm{CH}_{3} \mathrm{O}\right), 20.9(2 \mathrm{C}), 20.7(3 \mathrm{C}), 20.6$, and $20.4(7$ $\mathrm{COCH}_{3}$ ).

Methyl 4-O-acetyl-3-O-(2,3,4,6-tetra-O-acetyl- $\alpha$-D-mannopyranosyl)-6-O-(2,4,6tri-O-acetyl-3-O-methyl- $\alpha$-D-mannopyranosyl)-2-O-(2,3,4-tri-O-acetyl- $\beta$-D-xylopyranosyl)- $\beta$-D-mannopyranoside (33). - To a solution of $32(95 \mathrm{mg}, 0.12 \mathrm{mmol}$ ) and 2,3,4,6-tetra- $O$-acetyl- $\alpha$-D-mannopyranosyl trichloroacetimidate ${ }^{8}(27 ; 65 \mathrm{mg}, 0.13$ mmol) in $\mathrm{CH}_{2} \mathrm{Cl}_{2}(10 \mathrm{~mL})$ containing powdered $4 \mathrm{~A}$ molecular sieves $(1 \mathrm{~g})$ was added dropwise a solution of trimethylsilyl triflate $(50 \mu \mathrm{L})$ in $\mathrm{CH}_{2} \mathrm{Cl}_{2}(5 \mathrm{~mL})$ at $-40^{\circ} \mathrm{C}$, and the mixture was stirred for $1.5 \mathrm{~h}$. TLC $\left(9: 1 \mathrm{CH}_{2} \mathrm{Cl}_{2}\right.$-acetone) then indicated the disappearance of 32 and a new product $33\left(R_{f} 0.41\right)$, and pyridine (1 $\mathrm{mL}$ ) was added. The mixture was filtered through Celite and concentrated, and toluene $(3 \times 10 \mathrm{~mL})$ was evaporated from the residue. Column chromatography (85: $15 \mathrm{CH}_{2} \mathrm{Cl}_{2}$-acetone) of the residue gave 33 , isolated as a white solid $(92 \mathrm{mg}$, $70 \%) ;[\alpha]_{\mathrm{D}}-27^{\circ}(c \mathrm{c}) ; R_{f}$ 0.31. NMR data $\left(\mathrm{CDCl}_{3}\right):{ }^{1} \mathrm{H}, \delta 5.421$ and $5.042(2 \mathrm{dd}$, each $\left.1 \mathrm{H}, J_{2^{\prime \prime}, 3^{\prime \prime}}=J_{2^{\prime \prime \prime}, 3^{\prime \prime \prime}}=3.4 \mathrm{~Hz}, \mathrm{H}-2^{\prime \prime}, 2^{\prime \prime \prime}\right), 5.401\left(\mathrm{dd}, 1 \mathrm{H}, J_{3^{\prime \prime \prime}, 4^{\prime \prime \prime}} 10.0 \mathrm{~Hz}, \mathrm{H}-3^{\prime \prime \prime}\right)$, 4.949 and $4.816\left(2 \mathrm{~d}\right.$, each $\left.1 \mathrm{H}, J_{1^{\prime \prime}, 2^{\prime \prime}}=J_{1^{\prime \prime}, 2^{\prime \prime \prime}}=1.7 \mathrm{~Hz}, \mathrm{H}-1^{\prime \prime}, 1^{\prime \prime \prime}\right), 4.921(\mathrm{~m}, 1 \mathrm{H}$, $\left.J_{4^{\prime}, 3^{\prime}}=J_{4^{\prime}, 5^{\prime} a x}=7.1, J_{4^{\prime}, 5^{\prime} e q} 4.4 \mathrm{~Hz}, \mathrm{H}-4^{\prime}\right), 4.873\left(\mathrm{~d}, 1 \mathrm{H}, J_{1^{\prime}, 2^{\prime}} 5.8 \mathrm{~Hz}, \mathrm{H}-1^{\prime}\right), 4.365(\mathrm{~s}$, $1 \mathrm{H}, \mathrm{H}-1), 4.168\left(\mathrm{~d}, 1 \mathrm{H}, J_{2,3} 4.0 \mathrm{~Hz}, \mathrm{H}-2\right), 3.494$ and $3.398\left(2 \mathrm{~s}\right.$, each $3 \mathrm{H}, 2 \mathrm{CH}_{3} \mathrm{O}$ ), 2.144, 2.140, 2.123, 2.094, 2.089, 2.077, 2.044, 2.039, and $2.004(9 \mathrm{~s}, 3,3,3,3,9,3,3,3,3$ $\mathrm{H}, 11 \mathrm{Ac}) ;{ }^{13} \mathrm{C}, \delta 170.6-168.7\left(\mathrm{COCH}_{3}\right), 101.4,100.5,99.3$, and $97.5\left(\mathrm{C}-1,1^{\prime}, 1^{\prime \prime}, 1^{\prime \prime \prime}\right)$, $62.5(3 \mathrm{C})$ and $61.0\left(\mathrm{C}-6,5^{\prime}, 6^{\prime \prime}, 6^{\prime \prime \prime}\right), 57.8$ and $56.7\left(2 \mathrm{CH}_{3} \mathrm{O}\right), 20.9-20.5\left(\mathrm{COCH}_{3}\right)$. Anal. Calcd for $\mathrm{C}_{47} \mathrm{H}_{66} \mathrm{O}_{31}: \mathrm{C}, 50.09 ; \mathrm{H}, 5.90$. Found: $\mathrm{C}, 50.31 ; \mathrm{H}, 6.04$.

Methyl 3-O- $\alpha$-D-mannopyranosyl-6-O-(3-O-methyl- $\alpha$-D-mannopyranosyl)-2-O$\beta$-D-xylopyranosyl- $\beta$-D-mannopyranoside (4).-Deacetylation of 33 (40 $\mathrm{mg}, 36$ $\mu \mathrm{mol}$ ), as described for 2 , gave 4 , isolated as a white powder (24 $\mathrm{mg}, 96 \%) ;[\alpha]_{\mathrm{D}}$ $+4^{\circ}\left(c \quad 0.5, \mathrm{H}_{2} \mathrm{O}\right) ; R_{f} 0.46\left(2: 1: 1\right.$ 1-butanol-MeOH-water). ${ }^{13} \mathrm{C}$ NMR data 
$\left(\mathrm{D}_{2} \mathrm{O}\right): \delta 106.3,103.3,102.5$, and $100.8\left(\mathrm{C}-1,1^{\prime}, 1^{\prime \prime}, 1^{\prime \prime \prime}\right), 58.2$ and $57.6\left(2 \mathrm{CH}_{3} \mathrm{O}\right)$. For ${ }^{1} \mathrm{H}$ NMR data, see Table I.

\section{ACKNOWLEDGMENTS}

This investigation was supported by the Netherlands Foundation for Chemical Research (SON) with financial aid from the Netherlands Organization for Scientific Research (NWO). We thank Dr. A.M.P. van Steijn for the recording of several ${ }^{13} \mathrm{C}$ NMR spectra.

\section{REFERENCES}

1 J.A. van Kuik, R.P. Sijbesma, J.P. Kamerling, J.F.G. Vliegenthart, and E.J. Wood, Eur. J. Biochem., 160 (1986) 621-625.

2 J.A. van Kuik, R.P. Sijbesma, J.P. Kamerling, J.F.G. Vliegenthart, and E.J. Wood, Eur. J. Biochem., 169 (1987) 399-411.

3 G.Z. Lederkremer and A.J. Parodi, J. Biol. Chem., 259 (1984) 12514-12518.

4 J.P. Kamerling, Pure Appl. Chem., 63 (1991) 465-472.

5 J.B. Bouwstra, J. Kerékgyártó, J.P. Kamerling, and J.F.G. Vliegenthart, Carbohydr. Res., 186 (1989) 39-49.

6 B.R. Leeflang, J. Kerékgyártó, J.P. Kamerling, and J.F.G. Vliegenthart, Carbohydr. Res., 208 (1990) 117-126.

7 H. Mulder, H. Schachter, M. de Jong-Brink, J.G.M. van der Ven, J.P. Kamerling, and J.F.G. Vliegenthart, Eur. J. Biochem., 210 (1991) 459-465.

8 J. Kerékgyártó, J.P. Kamerling, J.B. Bouwstra, J.F.G. Vliegenthart, and A. Lipták, Carbohydr. Res., 186 (1989) 51-62.

9 J. Kerćkgyártó, J.G.M. van der Ven, J.P. Kamerling, A. Lipták, and J.F.G. Vlicgenthart, Carbohydr. Res., 238 (1993) 135-145.

10 H. Paulsen, Angew. Chem. Int. Ed. Engl., 21 (1982) 155-173.

11 P.A. Finan and C.D. Warren, J. Chem. Soc., (1962) 3089-3092.

12 R.F. Butterworth and S. Hanessian, Synthesis, (1971) 70-88.

13 P.M. Pojer and S.J. Angyal, Aust. J. Chem., 31 (1978) 1031-1040.

14 M. Mori, Y. Ito, and T. Ogawa, Carbohydr. Res., 191 (1990) 199-224.

15 M. Ek, P.J. Garegg, H. Hultberg, and S. Oscarson, J. Carbohydr. Chem., 2 (1983) 305-311.

16 A.K. Sen, K.K. Sarkar, and N. Banerji, J. Carbohydr. Chem., 7 (1988) 645-654.

17 S. Sato, Y. Ito, T. Nukada, Y. Nakahara, and T. Ogawa, Carbohydr. Res., 167 (1987) 197-210.

18 P.J. Garegg, H. Hultberg, and S. Oscarson, J. Chem. Soc., Perkin Trans. 1, (1982) 2395-2397.

19 E.J. Corey and J.W. Suggs, J. Org. Chem., 38 (1973) 3224.

20 O. Kanie, T. Takeda, N. Hada, and Y. Ogihara, J. Carbohydr. Chem., 10 (1991) 561-581.

21 C.V. Holland, D. Hortun, and J.S. Jewell, J. Org. Chem., 32 (1967) 1818-1821.

22 H. Paulsen, F. Garrido Espinose, W.P. Trautwein, and K. Heyns, Chem. Ber, 101 (1968) 179-185.

23 L.D. Hall and J.F. Manville, Carbohydr. Res., 4 (1967) 512-515.

24 A. de Bruyn, M. Anteunis, R. van Rijsbergen, M. Claeyssens, and $\mathrm{P}_{\urcorner}-$Kovác, J. Carbohydr. Chem., 1 (1982/1983) 301-309.

25 P.L. Durette, D. Horton, and N.S. Bhacca, Carbohydr. Res., 10 (1969) 565-577.

26 P.L. Durette and D. Horton, Carbohydr. Res., 18 (1971) 57-80.

27 E.E. Lee, G. Keaveney, and P.S. O'Colla, Carbohydr. Res., 59 (1977) 268-273. 